\title{
Acoustic Modes in a Ducted Shear Flow
}

\author{
Gregory Vilenski* \\ Sjoerd W. Rienstra ${ }^{\dagger}$ \\ Eindhoven University of Technology, 5600 MB Eindhoven, The Netherlands.
}

\begin{abstract}
The propagation of small-amplitude modes in an inviscid but sheared mean flow inside a duct is considered. For isentropic flow in a circular duct with zero swirl and constant mean flow density the pressure modes are described in terms of the eigenvalue problem for the Pridmore-Brown equation. A numerical method similar to the procedure used by Tam \& Auriault is proposed for the solution of the modal equation. Since for sufficiently high Helmholtz and wavenumbers, which are of great interest for the applications, the field equation is inherently stiff, special care is taken to insure the stability of the numerical algorithm designed to tackle this problem. The accuracy of the method is checked against the well-known analytical solution for the uniform flow. The numerical method is shown to be consistent with the analytical predictions at least for the Helmholtz numbers up to 100 and the circumferential wavenumber as large as 50, typical Mach numbers being up to 0.65 .

In order to gain further insight into the possible structure of the modal solutions and to get an independent verification of the robustness of the numerical scheme, the asymptotic solution of the problem based on the WKB method is derived. The comparisons of the WKB solution against the exact potential flow solution show remarkably good agreement between the two. This permits us to use the asymptotic solution as a benchmark for computations with high Helmholtz numbers, where numerical solutions of other authors are not available.

Numerical analysis of the problem with zero mean flow at the wall and acoustic lining shows that IngardMyers condition is recovered for vanishing boundary-layer thickness, although the boundary layer must be very thin in some cases.
\end{abstract}

\section{Nomenclature}

$\begin{array}{ll}t, x, r, \theta & =\text { time, axial, radial and circumferential coordinates } \\ u, v, w & =\text { projections of the velocity vector on the coordinate axes } x, r \text { and } \theta \\ \rho, p & =\text { density and pressure } \\ s & =\text { tip-to-hub ratio } \\ h & =\text { dimensional inner duct radius } \\ d & =\text { dimensional outer duct radius } \\ \omega & =\text { dimensional frequency } \\ m & =\text { circumferential wavenumber } \\ k & =\text { dimensional lateral wavenumber } \\ M & =\text { Mach number } \\ \sim & =\text { upper-case symbol denoting nondimensional values } \\ c_{0} & =\text { sound speed in the flow without swirl } \\ a_{0} & =\text { sound speed in swirling flow } \\ n & =\text { eigenmode number }\end{array}$

${ }^{*}$ Research Assistant, Department of Mathematics \& Computer Science, Eindhoven University of Technology, P.O. Box 513, 5600 MB Eindhoven, The Netherlands. AIAA Member.

${ }^{\dagger}$ Associate Professor, Department of Mathematics \& Computer Science, Eindhoven University of Technology, P.O. Box 513, 5600 MB Eindhoven, The Netherlands. AIAA Member. 


\section{Introduction}

ORMAL mode analysis of small-amplitude disturbances in an annular duct with an appropriately chosen mean 1 flow has been much used in the problems of turbomachinery noise propagation. Although an engine duct is not straight, the representation of sound in a duct by modes is very advantageous because of the clarity and the theoretical insight that it provides. The earlier analyses of this type (e.g. Zorumski [1]) used uniform flow, in which case acoustic modes can be studied independently from the hydrodynamic modes. By using the multiple scales technique for slowly varying ducts it is possible to extend the modal approach even further (Rienstra [2,3] Cooper \& Peake [4]).

Although the mean flow in the inlet duct is almost uniform with vorticity concentrated in the thin boundary layer, the mean flow of the by-pass duct is strongly sheared, sometimes with swirl, which requires more precise modelling. Since in the latter case the acoustic disturbances are not irrotational any more and are coupled with the hydrodynamic disturbances due to entropy and vorticity waves, the analysis of small amplitude modes becomes much more involved both computationally and theoretically. An excellent survey of the vast amount of the literature existing on the issue can be found in the article by Eversman [5]. More recently the problem of small-amplitude perturbations in swirling mean flows was considered in the works by Golubev \& Atassi [6], Tam \& Auriault [7], Nijboer [8], Cooper \& Peake [9], Kousen [10], etc.

The aim of this work is to revisit the problem of the propagation of small disturbances in an annular duct with sheared mean flow and wall lining. The reasons for this are as follows. Firstly, for sufficiently high Helmholtz and wavenumbers, which are of the most interest for the applications, the field equation is inherently stiff. This means that numerical stability of the algorithms designed to tackle this problem becomes a major issue to be taken into account. Secondly, even for hard walled ducts only limited data on the properties of the spectrum of this equation currently exists in the literature and only for relatively small values of the Helmholtz number. In particular, coverage of the properties of the hydrodynamic part of the spectrum, in our view, remains insufficient. Also, the influence of the wall lining over the spectrum structure and the range of validity of Myers condition as a limiting model for the mean flow velocity profiles with strong near-wall shear due to no-slip condition are not fully understood.

In order to gain further insight into these issues, we will develop a robust numerical method, valid for very high Helmholtz numbers. Its validity will be verified against asymptotic results of the WKB type similar to what has been proposed by Envia [11] and Cooper \& Peake [9].

The properties of the hydrodynamic part of the spectrum for the sheared mean flow without swirl (i.e. PridmoreBrown equation ${ }^{12}$ ) will be studied both analytically and numerically. In particular, the asymptotic analysis will show that for sheared mean flow with non-zero wall velocity and in the absence of lining the number of hydrodynamic modes is finite and they are localised near the duct walls. This result will be confirmed numerically. In parallel with this, the WKB solution will be extended to the case of soft walls. It will be used to predict analytically typical behaviour of the axial wavenumber in the complex plane when the wall impedance varies in the complex plain.

\section{Governing equations}

Consider an inviscid non-heat-conducting compressible perfect gas flow inside an infinitely long straight annular duct of inner radius $h$ and outer radius $d$. Let $x, r$ and $\theta$ be the axial, the radial and the circumferential coordinates, $u, v$ and $w$ the projections of the velocity vector on the coordinate axes $x, r$ and $\theta$ respectively, $\rho$ and $p$ the density and the pressure. The dimensional equations for conservation of mass, radial, circumferential, lateral components of momentum and energy are

$$
\begin{aligned}
\frac{\partial \rho}{\partial t}+\frac{1}{r} \frac{\partial(r \rho v)}{\partial r}+\frac{1}{r} \frac{\partial(\rho w)}{\partial \theta}+\frac{1}{r} \frac{\partial(\rho u)}{\partial x} & =0 \\
\frac{\partial v}{\partial t}+v \frac{\partial v}{\partial r}+\frac{w}{r} \frac{\partial v}{\partial \theta}+u \frac{\partial v}{\partial x}-\frac{w^{2}}{r} & =-\frac{1}{\rho} \frac{\partial p}{\partial r} \\
\frac{\partial w}{\partial t}+v \frac{\partial w}{\partial r}+\frac{w}{r} \frac{\partial w}{\partial \theta}+u \frac{\partial w}{\partial x}+\frac{v w}{r} & =-\frac{1}{r \rho} \frac{\partial p}{\partial \theta} \\
\frac{\partial u}{\partial t}+v \frac{\partial u}{\partial r}+\frac{w}{r} \frac{\partial u}{\partial \theta}+u \frac{\partial u}{\partial x} & =-\frac{1}{\rho} \frac{\partial p}{\partial x} \\
\frac{\partial p}{\partial t}+v \frac{\partial p}{\partial r}+\frac{w}{r} \frac{\partial p}{\partial \theta}+u \frac{\partial p}{\partial x}+\gamma p\left(\frac{1}{r} \frac{\partial(r v)}{\partial r}+\frac{1}{r} \frac{\partial w}{\partial \theta}+\frac{\partial u}{\partial x}\right) & =0
\end{aligned}
$$


Here $t$ is time, $\gamma=c_{p} / c_{v}$ is the ratio of specific heat capacities at constant pressure and constant volume, respectively. The pressure, the density and the absolute temperature $T$ satisfy the equation of state $p=R \rho T, R=c_{p}-c_{v}$. The energy equation (5) can be also written in terms of the entropy $S=c_{v} \ln \left(p / \rho^{\gamma}\right)+$ const in the following equivalent form

$$
\frac{\mathrm{d} S}{\mathrm{~d} t} \equiv \frac{\partial S}{\partial t}+v \frac{\partial S}{\partial r}+\frac{w}{r} \frac{\partial S}{\partial \theta}+u \frac{\partial S}{\partial x}=0 .
$$

Assume that the total flow field is the sum of a mean base flow and small-amplitude unsteady perturbations

$$
(u, v, w, \rho, p)=(\bar{u}, \bar{v}, \bar{w}, \bar{\rho}, \bar{p})+(\widetilde{u}, \widetilde{v}, \widetilde{w}, \tilde{\rho}, \widetilde{p}) .
$$

The mean flow is independent of $x$, its radial velocity is zero, its circumferential velocity is also independent of $\theta$, i.e.,

$$
\frac{\partial}{\partial x} \equiv 0, \bar{v}=0, \frac{\partial \bar{w}}{\partial \theta}=0
$$

In this case the mean flow is governed by the following system of equations

$$
\frac{\partial \bar{\rho}}{\partial t}+\frac{\bar{w}}{r} \frac{\partial \bar{\rho}}{\partial \theta}=0, \quad \frac{\bar{w}^{2}}{r}=\frac{1}{\bar{\rho}} \frac{\partial \bar{p}}{\partial r}, \quad \frac{\partial \bar{w}}{\partial t}+\frac{1}{r \bar{\rho}} \frac{\partial \bar{p}}{\partial \theta}=0, \quad \frac{\partial \bar{u}}{\partial t}+\frac{\bar{w}}{r} \frac{\partial \bar{u}}{\partial \theta}=0, \quad \frac{\partial \bar{p}}{\partial t}+\frac{\bar{w}}{r} \frac{\partial \bar{p}}{\partial \theta}=0 .
$$

which has solution

$$
\bar{\rho}=\bar{\rho}(r), \quad \bar{w}=\bar{w}(r), \quad \bar{u}=\bar{u}\left(r, \theta-\frac{\bar{w}(r)}{r} t\right), \quad p=p_{d}-\int_{r}^{d} \bar{\rho}(\xi) \frac{\bar{w}^{2}(\xi)}{\xi} \mathrm{d} \xi, \quad \bar{v}=0,
$$

where $p_{d}=$ const is the pressure at $r=d, \bar{\rho}(r), \bar{w}(r)$ are arbitrary functions of $r$, and $\bar{u}$ is an arbitrary function of its arguments.

In turbomachinery context, (7) could be used as a simplified time-dependent model for the wake flow behind a fan. If the fan rotates with an angular velocity $\widetilde{\Omega}$ and $\theta^{\prime}$ is the circumferential angle in the rotating frame so that $\theta=\widetilde{\Omega} t+\theta^{\prime}$, then for the rotor with $l$ blades the wake profile $\bar{u}=\bar{u}\left(r, \theta^{\prime}+\frac{\widetilde{\Omega} r-\bar{w}(r)}{r} t\right)$ must be a periodic function in $\theta^{\prime}$ with the period $2 \pi / l$, or after Fourier decomposition in $\theta^{\prime}$

$$
\bar{u}=\sum_{k=-\infty}^{\infty} \bar{u}_{k}(r) e^{i k l\left(\theta^{\prime}+\frac{\tilde{\Omega} r-\bar{w}(r)}{r} t\right)}=\sum_{k=-\infty}^{\infty} \bar{u}_{k}(r) e^{i k l\left(\theta-\frac{\bar{w}(r)}{r} t\right)} .
$$

All three vorticity components are non-zero for the mean flow (7)

$$
\Omega_{x}=\frac{1}{r} \frac{\mathrm{d}(r \bar{w})}{\mathrm{d} r}, \quad \Omega_{\theta}=-\frac{\partial \bar{u}}{\partial r}, \quad \Omega_{r}=\frac{1}{r} \frac{\partial \bar{u}}{\partial \theta} .
$$

If the mean flow is time-independent, (7) reduces to the following well known mean flow solution (see for instance, Tam \& Auriault [7])

$$
\bar{u}=\bar{u}(r), \quad \bar{v}=0, \quad \bar{w}=\bar{w}(r), \quad \bar{\rho}=\bar{\rho}(r), \quad \bar{p}=p_{d}-\int_{r}^{d} \bar{\rho}(\xi) \frac{\bar{w}^{2}(\xi)}{\xi} \mathrm{d} \xi
$$

with the swirl $\Omega_{x}=\mathrm{d} \bar{w} / \mathrm{d} r+\bar{w} / r$, the circumferential vorticity (shear) $\Omega_{\theta}=-\mathrm{d} \bar{u} / \mathrm{d} r$, and zero radial vorticity.

For the mean flow (7) small-amplitude disturbance field is governed by the linearized Euler equations

$$
\begin{aligned}
\frac{\partial \widetilde{\rho}}{\partial t}+\bar{u} \frac{\partial \widetilde{\rho}}{\partial x}+\frac{1}{r} \frac{\partial(r \bar{\rho} \widetilde{v})}{\partial r}+\frac{\bar{w}}{r} \frac{\partial \widetilde{\rho}}{\partial \theta}+\bar{\rho}\left(\frac{1}{r} \frac{\partial \widetilde{w}}{\partial \theta}+\frac{\partial \widetilde{u}}{\partial x}\right) & =0, \\
\bar{\rho}\left(\frac{\partial \widetilde{v}}{\partial t}+\bar{u} \frac{\partial \widetilde{v}}{\partial x}+\frac{\bar{w}}{r} \frac{\partial \widetilde{v}}{\partial \theta}-\frac{2 \bar{w} \widetilde{w}}{r}\right)-\widetilde{\rho} \frac{\bar{w}^{2}}{r} & =-\frac{\partial \widetilde{p}}{\partial r}, \\
\bar{\rho}\left(\frac{\partial \widetilde{w}}{\partial t}+\bar{u} \frac{\partial \widetilde{w}}{\partial x}+\frac{\bar{w}}{r} \frac{\partial \widetilde{w}}{\partial \theta}+\widetilde{v}\left(\frac{\mathrm{d} \bar{w}}{\mathrm{~d} r}+\frac{\bar{w}}{r}\right)\right) & =-\frac{1}{r} \frac{\partial \widetilde{p}}{\partial \theta}, \\
\bar{\rho}\left(\frac{\partial \widetilde{u}}{\partial t}+\bar{u} \frac{\partial \widetilde{u}}{\partial x}+\frac{\bar{w}}{r} \frac{\partial \widetilde{u}}{\partial \theta}+\widetilde{v} \frac{\partial \bar{u}}{\partial r}+\frac{\widetilde{w}}{r} \frac{\partial \bar{u}}{\partial \theta}\right) & =-\frac{\partial \widetilde{p}}{\partial x}, \\
\frac{\partial \widetilde{p}}{\partial t}+\bar{u} \frac{\partial \widetilde{p}}{\partial x}+\frac{\bar{w}}{r} \frac{\partial \widetilde{p}}{\partial \theta}+\widetilde{v} \bar{\rho} \frac{\bar{w}^{2}}{r}+\gamma \bar{p}\left(\frac{1}{r} \frac{\partial(r \widetilde{v})}{\partial r}+\frac{1}{r} \frac{\partial \widetilde{w}}{\partial \theta}+\frac{\partial \widetilde{u}}{\partial x}\right) & =0 .
\end{aligned}
$$


Assume in what follows that the mean flow is time- and $\theta$-independent and is given by the relations (8). The resulting small-amplitude disturbances are sought in the form

$$
(\widetilde{u}, \widetilde{v}, \widetilde{w}, \widetilde{\rho}, \widetilde{p})=(U, V, W, R, P) \exp (-i \omega t+i k x+i m \theta)
$$

where $\omega$ is the excitation frequency, $k$ and $m$ are the lateral and the circumferential wavenumbers, respectively. The amplitudes $(U, V, W, R, P)$ are unknown functions of $r$. They satisfy the system of equations

$$
\begin{aligned}
i \lambda R+\frac{1}{r} \frac{\mathrm{d}(r \bar{\rho} V)}{\mathrm{d} r}+\bar{\rho}\left(\frac{i m}{r} W+i k U\right) & =0, \\
i \lambda V-\frac{2 \bar{w}}{r} W-\frac{\bar{w}^{2}}{\bar{\rho} r} R & =-\frac{1}{\bar{\rho}} P^{\prime}, \\
i \lambda W+\left(\bar{w}^{\prime}+\frac{\bar{w}}{r}\right) V & =-\frac{i m}{\bar{\rho} r} P, \\
i \lambda U+\bar{u}^{\prime} V & =-\frac{i k}{\bar{\rho}} P, \\
i \lambda P+\frac{\bar{w}^{2} \bar{\rho}}{r} V+\gamma \bar{p}\left(\frac{1}{r} \frac{\mathrm{d}(r V)}{\mathrm{d} r}+\frac{i m}{r} W+i k U\right) & =0 .
\end{aligned}
$$

Here and in what follows the primes denote differentiation with respect to $r, \lambda=-\omega+k \bar{u}+m \bar{w} / r$ is the eigenvalue of the operator $-i\left(\frac{\partial}{\partial t}+\bar{u} \frac{\partial}{\partial x}+\frac{\bar{w}}{r} \frac{\partial}{\partial \theta}\right)$. In order to reduce system (15-19) to a single equation, we first eliminate $U$ and $W$. This can be achieved by eliminating $W$ from (16) and(17), $\frac{i m}{r} W+i k U$ - from (15) and (19), expressing the latter sum via (17) and (18) and substituting it into (15):

$$
\begin{aligned}
& i \lambda \bar{\rho} P-i \lambda \gamma \bar{p} R+\left(\frac{\bar{w}^{2}}{r} \bar{\rho}^{2}-\gamma \bar{\rho}^{\prime} \bar{p}\right) V=0, \\
& \frac{2 i m \bar{w}}{\bar{\rho} r^{2}} P-\frac{i \lambda \bar{w}^{2}}{\bar{\rho} r} R+\left(\frac{2 \bar{w}}{r}\left(\bar{w}^{\prime}+\frac{\bar{w}}{r}\right)-\lambda^{2}\right) V=-\frac{i \lambda}{\bar{\rho}} P^{\prime}, \\
& i \lambda R+\frac{1}{r} \frac{\mathrm{d}(r \bar{\rho} V)}{\mathrm{d} r}+\frac{1}{i \lambda}\left(\frac{m^{2}}{r^{2}}+k^{2}\right) P-\frac{\bar{\rho}}{\lambda}\left(\frac{m}{r}\left(\bar{w}^{\prime}+\frac{\bar{w}}{r}\right)+k \bar{u}^{\prime}\right) V=0 .
\end{aligned}
$$

Eliminating $R$ from (21) and (22) via (20) yields the system for the pressure and radial velocity amplitudes

$$
\begin{aligned}
\frac{i \lambda P^{\prime}}{\bar{\rho}}+B \frac{i P}{\bar{\rho}}-A V & =0, \\
\frac{1}{r} \frac{\mathrm{d}(r V)}{\mathrm{d} r}+\frac{i \Omega}{\bar{\rho} \lambda} P+C V & =0 .
\end{aligned}
$$

Here

$$
\begin{gathered}
A=\lambda^{2}+\frac{\bar{w}^{4}}{\bar{a}^{2} r^{2}}-\frac{\bar{w}^{2}}{r} \frac{\bar{\rho}^{\prime}}{\bar{\rho}}-\frac{2 \bar{w}}{r}\left(\bar{w}^{\prime}+\frac{\bar{w}}{r}\right), \quad B=\frac{2 m \bar{w}}{r^{2}}-\frac{\lambda \bar{w}^{2}}{\bar{a}^{2} r}, \\
C=\frac{\bar{w}^{2}}{\bar{a}^{2} r}-\frac{1}{\lambda}\left(\frac{m}{r}\left(\bar{w}^{\prime}+\frac{\bar{w}}{r}\right)+k \bar{u}^{\prime}\right), \quad \Omega=\frac{\lambda^{2}}{\bar{a}^{2}}-k^{2}-\frac{m^{2}}{r^{2}}, \quad \bar{a}^{2}=\frac{\gamma \bar{p}}{\bar{\rho}} .
\end{gathered}
$$

System (23-24) coincides with the related system of equations obtained in the work by Nijboer [8]. Elimination of $V$ leads to a single differential equation for the pressure amplitude $P$

$$
P^{\prime \prime}+\left(\frac{\lambda+B r}{\lambda r}+C+\frac{\bar{\rho} A}{\lambda} \frac{\mathrm{d}}{\mathrm{d} r}\left(\frac{\lambda}{\bar{\rho} A}\right)\right) P^{\prime}+\left(\frac{A \Omega}{\lambda^{2}}+\frac{\bar{\rho} A}{\lambda r} \frac{\mathrm{d}}{\mathrm{d} r}\left(\frac{B r}{\bar{\rho} A}\right)+\frac{B C}{\lambda}\right) P=0 .
$$

In order to state boundary conditions for this equation, assume that the duct walls are treated with locally reacting lining with the complex specific impedances $Z_{h}$ and $Z_{d}$ for $r=h$ and $r=d$, respectively. According to Ingard [13] and Myers [14], the following relations must be satisfied on the duct walls

$$
-i \omega \widetilde{v}_{n}=\left(-i \omega+\bar{u} \frac{\partial}{\partial x}+\frac{\bar{w}}{r} \frac{\partial}{\partial \theta}\right)\left(\frac{\tilde{p}}{Z}\right)
$$


where $\widetilde{v}_{n}$ is the projection of the perturbation velocity on the outwardly directed normal to the duct wall ( $\widetilde{v}_{n}=$ $\widetilde{v}$ for $r=d$ and $\widetilde{v}_{n}=-\widetilde{v}$ for $r=h$ ), $Z=Z_{d}$ or $Z=Z_{h}$ for $r=d$ or $r=h$, respectively. Substitution of (14) in (26) together with (23) gives the required boundary conditions for the equation (25)

$$
\begin{aligned}
& P^{\prime}+\left(\frac{B}{\lambda}+\frac{i \bar{\rho} A}{\omega Z_{h}}\right) P=0 \quad \text { on } r=h, \\
& P^{\prime}+\left(\frac{B}{\lambda}-\frac{i \bar{\rho} A}{\omega Z_{d}}\right) P=0 \quad \text { on } r=d .
\end{aligned}
$$

The hard wall boundary condition on either of the walls is recovered in the limit $Z_{h}$ or $Z_{d} \rightarrow \infty$.

In the eigenvalue problem $(25,27,28)$ the excitation frequency $\omega$ and the circumferential wavenumber $m$ are the given numbers while the lateral wavenumber $k$ is an unknown spectral parameter. In the special case of zero mean circumferential velocity $\bar{w}=0$ and constant mean flow density $\bar{\rho}$ (and hence the pressure) (25) reduces to the PridmoreBrown equation

$$
P^{\prime \prime}+\beta(r) P^{\prime}+\gamma(r) P=0
$$

where

$$
\beta(r)=\left(\frac{1}{r}+\frac{2 k \bar{u}^{\prime}}{\omega-k \bar{u}}\right), \quad \gamma(r)=\left(\frac{(\omega-k \bar{u})^{2}}{c_{0}^{2}}-k^{2}-\frac{m^{2}}{r^{2}}\right) \quad \text { and } c_{0}^{2}=\frac{\gamma p_{d}}{\bar{\rho}} .
$$

In this case the boundary conditions are

$$
\begin{aligned}
& P^{\prime}+\frac{i \bar{\rho}(\omega-k \bar{u})^{2}}{\omega Z_{h}} P=0 \quad \text { on } r=h, \\
& P^{\prime}-\frac{i \bar{\rho}(\omega-k \bar{u})^{2}}{\omega Z_{d}} P=0 \quad \text { on } r=d .
\end{aligned}
$$

The present work studies the solutions of field equation (29) with various versions of conditions $(30,31)$. The exception is the numerical procedure described below which was also used to integrate the generic problem $(25,27,28)$.

\section{Asymptotic analysis}

The following non-dimensional quantities will be used in this section

$$
\begin{gathered}
\tilde{\omega}=\frac{\omega d}{c_{0}}, \quad \tilde{k}=k d, \quad z_{h}=Z_{h} /\left(\bar{\rho} c_{0}\right), \quad z_{d}=Z_{d} /\left(\bar{\rho} c_{0}\right), \\
M=\bar{u} / c_{0}, \quad M_{h}=\bar{u}(h) / c_{0}, \quad M_{d}=\bar{u}(d) / c_{0}, \quad s=h / d .
\end{gathered}
$$

Also assume that the pressure amplitude $P$ is scaled by $\bar{\rho} c_{0}^{2}$, time by $d / c_{0}$, velocities by the sound speed $c_{0}, r$ and other distances by $d$, so that the problem (29-31) can be rewritten in the following non-dimensional form

$$
\begin{aligned}
& P^{\prime \prime}+\left(\frac{1}{r}+\frac{2 \widetilde{k} M^{\prime}}{\widetilde{\omega}-\widetilde{k} M}\right) P^{\prime}+\left((\widetilde{\omega}-\widetilde{k} M)^{2}-\widetilde{k}^{2}-\frac{m^{2}}{r^{2}}\right) P=0, \\
& P^{\prime}+\frac{i\left(\widetilde{\omega}-\widetilde{k} M_{h}\right)^{2}}{\widetilde{\omega} z_{h}} P=0 \quad \text { on } r=s(=h / d), \\
& P^{\prime}-\frac{i\left(\widetilde{\omega}-\widetilde{k} M_{d}\right)^{2}}{\widetilde{\omega} z_{d}} P=0 \quad \text { on } r=1 .
\end{aligned}
$$

In order to reduce the system (32-34) to the form convenient for WKB analysis, introduce new independent variable $\tau=\tau(r)$ which will be specified in what follows and new dependent variable $w(\tau)$ (not to be confused with the total circumferential velocity $w$ in section 2) such that

$$
P=\alpha(r)\left(\tau^{\prime}\right)^{-1 / 2} w(\tau), \quad \alpha(r)=\frac{\widetilde{\omega}-\widetilde{k} M}{\sqrt{r}}
$$

and, hence,

$$
P^{\prime}=\alpha(r)\left[\left(\tau^{\prime}\right)^{1 / 2} \frac{\mathrm{d} w}{\mathrm{~d} \tau}-\frac{1}{2} \tau^{\prime \prime}\left(\tau^{\prime}\right)^{-3 / 2} w\right]+\frac{\mathrm{d} \alpha}{\mathrm{d} r}\left(\tau^{\prime}\right)^{-1 / 2} w
$$


The system (32-34) then takes the following form

$$
\begin{gathered}
\frac{\mathrm{d}^{2} w}{\mathrm{~d} \tau^{2}}+\left\{\left(\tau^{\prime}\right)^{-2} q-\frac{1}{2} \tau^{\prime \prime \prime}\left(\tau^{\prime}\right)^{-3}+\frac{3}{4}\left(\tau^{\prime \prime}\right)^{2}\left(\tau^{\prime}\right)^{-4}\right\} w=0, \\
\frac{\mathrm{d} w}{\mathrm{~d} \tau}+\left\{-\frac{1}{2} \tau^{\prime \prime}\left(\tau^{\prime}\right)^{-1}+\frac{\alpha^{\prime}(s)}{\alpha(s)}+\frac{i\left(\widetilde{\omega}-\widetilde{k} M_{h}\right)^{2}}{\widetilde{\omega} z_{h}}\right\}\left(\tau^{\prime}\right)^{-1} w=0 \text { at } \tau=\tau(s), \\
\frac{\mathrm{d} w}{\mathrm{~d} \tau}+\left\{-\frac{1}{2} \tau^{\prime \prime}\left(\tau^{\prime}\right)^{-1}+\frac{\alpha^{\prime}(1)}{\alpha(1)}-\frac{i\left(\widetilde{\omega}-\tilde{k} M_{d}\right)^{2}}{\widetilde{\omega} z_{d}}\right\}\left(\tau^{\prime}\right)^{-1} w=0 \text { at } \tau=\tau(1),
\end{gathered}
$$

where

$$
q(r)=(\widetilde{\omega}-\widetilde{k} M)^{2}-\widetilde{k}^{2}-\frac{m^{2}}{r^{2}}-\frac{2 \widetilde{k}^{2}\left(M^{\prime}\right)^{2}}{(\widetilde{\omega}-\widetilde{k} M)^{2}}-\frac{\widetilde{k}\left(M^{\prime \prime}+M^{\prime} / r\right)}{\widetilde{\omega}-\widetilde{k} M}+\frac{1}{4 r^{2}} .
$$

It is assumed in this section that the duct is not hollow $(s>0)$, the spectral parameter $\tilde{k}$ is large and, unless specified otherwise, the frequency $\widetilde{\omega}$ and the circumferential wavenumber $m$ are of the same order of magnitude as $\widetilde{k}$. It follows from (39) that $q(r)=O\left(\widetilde{k}^{2}\right)$ as $\widetilde{k} \rightarrow \infty$. The exceptions are the vicinities of turning points, say $r=r_{*}$, where $q\left(r_{*}\right)=0$ and the vicinities of the points, where $\widetilde{\omega}-\widetilde{k} M$ goes to zero (Cooper \& Peake [9]). According to the WKB methodology (see for instance Barantsev \& Engelgart [15]), away from these points the main-order part of the operator on the left-hand side of (36) can be extracted by letting $\tau^{\prime}(r)=q^{1 / 2}$. In this case the first term in the curly parentheses is equal to one whereas the remaining terms are $O\left(\widetilde{k}^{-2}\right)$ and can be neglected in the main-order analysis. The case of one turning point can be dealt with by setting $\tau^{\prime}(r)=(q / \tau)^{1 / 2}$. The first term in the curly parentheses in (36) is then equal to $\tau=O\left(\widetilde{k}^{2 / 3}\right)$, with the other two terms being $O\left(\widetilde{k}^{-2 / 3}\right)$ uniformly in $r$, if $\widetilde{\omega}-\widetilde{k} M(r) \neq 0$. The case of $\widetilde{\omega}-\widetilde{k} M(r)=0$ will be studied separately. Although physically quite possible, the situation of multiple turning points will not be tackled in the present study.

\section{A. Ordinary WKB approximation}

Assume that $\widetilde{\omega}, m$ and $\widetilde{k}$ are such that neither $q(r)$ nor $\widetilde{\omega}-\tilde{k} M(r)$ go to zero for $s \leq r \leq d$ and let

$$
\tau(r)=\int_{s}^{r} \sqrt{q(\xi)} \mathrm{d} \xi, \quad \tau_{d}=\tau(1) \equiv \int_{s}^{1} \sqrt{q(\xi)} \mathrm{d} \xi .
$$

Then, provided that

$$
(\widetilde{\omega}-\widetilde{k} M)^{2} /(\widetilde{\omega} z) \gg 1
$$

for $z=z_{h}, z_{d}$, the problem (36-38) becomes

$$
\frac{\mathrm{d}^{2} w}{\mathrm{~d} \tau^{2}}+w=0, \quad \frac{\mathrm{d} w}{\mathrm{~d} \tau}+i \sigma_{h} w=0 \quad \text { at } \tau=0, \quad \frac{\mathrm{d} w}{\mathrm{~d} \tau}-i \sigma_{d} w=0 \quad \text { at } \tau=\tau_{d} .
$$

Here

$$
\sigma_{h}=\frac{\left(\widetilde{\omega}-\widetilde{k} M_{h}\right)^{2}}{\widetilde{\omega} z_{h} \tau^{\prime}(s)}, \quad \sigma_{d}=\frac{\left(\widetilde{\omega}-\widetilde{k} M_{d}\right)^{2}}{\widetilde{\omega} z_{d} \tau^{\prime}(1)}
$$

It has non-zero solution $w=\cos (\tau)-i \sigma_{h} \sin (\tau)$ if the spectral parameter $\widetilde{k}$ satisfies the equation

$$
e^{i 2 \tau_{d}}=\zeta, \quad \zeta \equiv \frac{1+\sigma_{h} \sigma_{d}+\sigma_{h}+\sigma_{d}}{1+\sigma_{h} \sigma_{d}-\sigma_{h}-\sigma_{d}}
$$

\section{Perturbations of the hard-wall solutions}

For the duct with hard walls $\sigma_{h}=\sigma_{d}=0$ the dispersion relation (43) reduces to the following equation for $\tilde{k}$

$$
\int_{s}^{1} \sqrt{q(\xi)} \mathrm{d} \xi=-n \pi,
$$

where $n$ is an integer number. 
It is interesting to compare the accuracy of WKB prediction of the lateral wavenumbers $\widetilde{k}$ based on the dispersion relation (44) with the well-known exact solution of system (32-34) for a uniform mean flow in a duct with hard walls. If $M^{\prime}$ is identically zero and $z_{h}=z_{d}=\infty$, the system (32-34) reduces to the boundary value problem for the Bessel equation

$$
\frac{\mathrm{d}^{2} P}{\mathrm{~d} r^{2}}+\frac{1}{r} \frac{\mathrm{d} P}{\mathrm{~d} r}+\left(\alpha^{2}-\frac{m^{2}}{r^{2}}\right) P=0, \quad r=s, 1: \frac{\mathrm{d} P}{\mathrm{~d} r}=0,
$$

with the dispersion relation being

$$
J_{m}^{\prime}(\alpha s) Y_{m}^{\prime}(\alpha)-Y_{m}^{\prime}(\alpha s) J_{m}^{\prime}(\alpha)=0, \quad \alpha=\sqrt{\frac{\omega^{2}}{c_{0}^{2}}-k^{2}},
$$

where the primes stand for derivatives of the Bessel functions with respect to their arguments.

The results of comparison between the WKB approximation (44) of the eigenvalues $\widetilde{k}$ and their exact counterparts based on the above formula are summarized in Table 1, which shows the first five complex eigenvalues for $\widetilde{\omega}=25$, $m=15, s=0.6, M=0.5$. It can be seen from Table 1 that apart from the first lateral wavenumber $\widetilde{k}$ with the smallest absolute value, for which the relative error is $3.6 \%$, the WKB prediction is very good, and the accuracy improves as $|\widetilde{k}|$ grows. This remarkable feature of the WKB approximation will be used in what follows to check the accuracy of the proposed numerical scheme for large $\widetilde{\omega}, m$, where the accuracy of the WKB approximation becomes even better whereas the accuracy of numerical schemes decreases due to the stiffness of the problem.

Table 1. Ordinary WKB approximation versus exact solution.

\begin{tabular}{rrr} 
WKB & $\begin{array}{c}\text { Exact } \\
k=16 .(6)+i .\end{array}$ & $\begin{array}{c}\text { Relative error } \\
k=16 .(6)+i .\end{array}$ \\
\hline 11.69 & 12.11 & 3.6 \\
26.62 & 26.73 & 0.41 \\
38.04 & 38.1 & 0.16 \\
48.49 & 48.53 & 0.082 \\
58.48 & 58.51 & 0.051
\end{tabular}

In general, the equations (43) or (44) must be solved numerically. One exception is the case of a slender duct. Then $\Delta=1-s \ll 1$ and

$$
\tau(r)=\sqrt{q(s)}(r-s)+\frac{q^{\prime}(s)}{\sqrt{q(s)}} \frac{(r-s)^{2}}{4}+O\left(\Delta^{3}\right), \quad s \leq r \leq 1,
$$

since it was assumed that $q(r) \neq 0$. Making use of the expansion $q(r)=(\widetilde{\omega}-\widetilde{k} M)^{2}-\widetilde{k}^{2}-m^{2} / r^{2}+O(1)$ as $\widetilde{k} \rightarrow \infty$ and taking $r=1$, yields

$$
q(s)=\left(\frac{\pi n}{\Delta}\right)^{2}-\frac{1}{2} q^{\prime}(s) \Delta+\ldots,
$$

or after some algebra

$$
\begin{gathered}
\tilde{k}=\frac{-0.5 \widetilde{\omega}\left(M_{h}+M_{d}\right) \pm \sqrt{D}}{1-M_{h} M_{d}} \\
D=\widetilde{\omega}^{2}-\left(1-M_{h} M_{d}\right)\left\{\left(\frac{m}{s}\right)^{2}(1-\Delta / s)+\left(\frac{\pi n}{\Delta}\right)^{2}\right\}+O\left(\Delta^{2}\right) .
\end{gathered}
$$

This solution is perfectly analogous to the uniform mean flow solution which is recovered when $M_{h}$ is set equal to $M_{d}$. The eigenvalues (45) are distributed symmetrically with respect to the real axis in the complex plane $\widetilde{k}$ and as $n$ goes to infinity with $\widetilde{\omega}$ and $m$ being fixed, $\widetilde{k}$ approaches the vertical line $\widetilde{k}=-0.5 \widetilde{\omega}\left(M_{h}+M_{d}\right) /\left(1-M_{h} M_{d}\right)$ (see also Figure 1).

According to the results recently reported by Rienstra [16] for the lined hollow duct with uniform mean flow, the problem for a lined duct may be expected to feature a much richer behaviour. Owing to the complicated character of the dispersion relation (43), further simplifying assumption $\widetilde{\omega} \approx|m| \ll|\widetilde{k}|$ as $\widetilde{k} \rightarrow \infty$ is needed to make this case 
analytically treatable. Also, assume that the inner wall is hard so that $\sigma_{h}=0$ and $\zeta=\left(1+\sigma_{d}\right) /\left(1-\sigma_{d}\right)$. Equation (43) then can be rewritten in the following form

$$
2 i \tau_{d}=-2 \pi n i+\ln \left(\frac{z_{d}+z_{0}}{z_{d}-z_{0}}\right), \quad \text { here } z_{0}=\frac{\left(\widetilde{\omega}-\widetilde{k} M_{d}\right)^{2}}{\widetilde{\omega} \sqrt{q(1)}},
$$

where $n$ is an integer. Note that for large $\widetilde{k}$ the logarithmic term in (46) is $O(1)$ when $z_{0} \neq \pm z_{d}$ whereas $\tau_{d}=O(\widetilde{k})$. Hence to leading order, the solution to the equation (46) is governed by the dispersion relation obtained above for the hard-wall boundary conditions $\tau_{d} \approx-\pi n$, where $|n|$ is large. The logarithmic term influences higher order terms. Expanding $z_{0}$ and $\tau_{d}$ in powers of $\tilde{k}$

$$
z_{0}=-i\left(\zeta_{1} \widetilde{k}-\zeta_{0}+O\left(\widetilde{k}^{-1}\right)\right), \quad \tau_{d}=i\left(\kappa_{1} \widetilde{k}+\kappa_{0}-\kappa_{-1} \widetilde{k}^{-1}+O\left(\widetilde{k}^{-2}\right)\right)
$$

and substituting these formulas in (46) gives

$$
\widetilde{k}+\frac{\kappa_{0}}{\kappa_{1}}-\frac{\kappa_{-1}}{\kappa_{1}} \widetilde{k}^{-1}+O\left(\widetilde{k}^{-2}\right)=\frac{\pi n i}{\kappa_{1}}-\frac{1}{2 \kappa_{1}} \ln \frac{z_{d}-i\left(\zeta_{1} \widetilde{k}-\zeta_{0}+O\left(\widetilde{k}^{-1}\right)\right)}{z_{d}+i\left(\zeta_{1} \tilde{k}-\zeta_{0}+O\left(\widetilde{k}^{-1}\right)\right)},
$$

where

$$
\begin{gathered}
\zeta_{1}=\frac{\widetilde{\omega}^{-1} M_{d}^{2}}{\sqrt{1-M_{d}^{2}}}, \quad \zeta_{0}=\frac{\left(2-M_{d}^{2}\right) M_{d}}{\left(1-M_{d}^{2}\right)^{3 / 2}}, \quad \kappa_{1}=\int_{s}^{1} \sqrt{1-M^{2}} \mathrm{~d} r, \quad \kappa_{0}=\int_{s}^{1} \frac{\widetilde{\omega} M}{\sqrt{1-M^{2}}} \mathrm{~d} r \\
\kappa_{-1}=\frac{1}{2} \int_{s}^{1}\left\{\frac{\widetilde{\omega}^{2}}{1-M^{2}}-\frac{m^{2}}{r^{2}}-\frac{2 M^{\prime 2}}{M^{2}}+\frac{M^{\prime \prime}+M^{\prime} / r}{M}+\frac{1}{4 r^{2}}\right\} \frac{\mathrm{d} r}{\sqrt{1-M^{2}}} .
\end{gathered}
$$

Consider at first the hard-wall limit $\left|z_{d}\right| \gg \zeta_{1} \pi n / \kappa_{1} \gg 1$ with the real and imaginary parts of the impedance $z_{d}$ being of the same order of magnitude. Since $|\widetilde{k}| \sim \pi n / \kappa_{1}$, the logarithmic term on the right-hand side of (47) can be further simplified, and the following solution for $\tilde{k}$ can be obtained

$$
\widetilde{k}=\left\{\frac{\pi n i}{\kappa_{1}}-\frac{\kappa_{0}}{\kappa_{1}}-\frac{i \kappa_{-1}}{\pi n} \ldots\right\}-\frac{1}{z_{d}}\left(\frac{\zeta_{1} \pi n}{\kappa_{1}^{2}}+\ldots\right) .
$$

Here the curly parentheses contains the expansion of the hard-wall solution in powers of $n$. The second term is the leading order correction due to the lining. Let $R$ and $X$ be the real and imaginary parts of the specific impedance $z_{d}$ and $\widetilde{k}_{r}$ and $\widetilde{k}_{i}$ - the real and imaginary part of the lateral wavenumber $\widetilde{k}$. Then elimination of $X$ from (48) yields the following relationship between $\widetilde{k}_{r}$ and $\widetilde{k}_{i}$

$$
\left(\widetilde{k}_{r}+\frac{\kappa_{0}}{\kappa_{1}}+\frac{\zeta_{1} \pi n}{2 R \kappa_{1}^{2}}\right)^{2}+\left(\widetilde{k}_{i}-\frac{\pi n}{\kappa_{1}}+\frac{\kappa_{-1}}{\pi n}\right)^{2}=\left(\frac{\zeta_{1} \pi n}{2 R \kappa_{1}^{2}}\right)^{2} .
$$

Expression (49) shows that when the real part $R$ of the impedance $z_{d}$ is fixed and its complex part $X$ is allowed to vary, the eigenvalues whose index $n$ is not too large i.e. $\left.\left|z_{d}\right| \gg \zeta_{1} \pi n / \kappa_{1} \gg 1\right)$ travel along the circle which surrounds the corresponding hard-wall eigenvalue $\widetilde{k}^{0}=\widetilde{k}_{r}^{0}+\widetilde{k}_{i}^{0}$, where

$$
\widetilde{k}_{r}^{0}=-\frac{\kappa_{0}}{\kappa_{1}}+\ldots, \quad \widetilde{k}_{i}^{0}=\frac{\pi n}{\kappa_{1}}-\frac{\kappa_{-1}}{\pi n}+\ldots
$$

Its radius decreases with the damping $R$ and the frequency $\widetilde{\omega}$ and grows with the Mach number and with the index $n$. The centre of the circle is shifted to the left from the position of the hard-wall eigenvalue $\widetilde{k}^{0}$ by the amount equal to its radius for positive $n$ and to the right - for negative $n$. The neighbouring circles are shifted from one another in the vertical direction by the amount $\pi / \kappa_{1}+O\left(1 / n^{2}\right)$ which depends mainly on the Mach number distribution and the hub-to-tip ratio $s$.

This result gives theoretical explanation for the appearance of circular trajectories in the complex plane of the lateral wavenumber for sufficiently large but fixed damping $R$ and varying $X$ which were obtained numerically in Rienstra [16] for a lined duct with constant mean flow (see also Figures 5a and 5b in what follows). Owing to the 
difference in the sign convention before the term $i \omega t$ in the above work and the present study, the direction of the horizontal shifts of the circles (49) is opposite to those in the paper by Rienstra [16].

Consider now a more general situation when $\left|z_{d}\right| \sim \zeta_{1} \pi n / \kappa_{1} \gg 1$, in which case it follows from (47) that

$$
\widetilde{k}=\widetilde{k}_{r}^{0}+i \widetilde{k}_{i}^{0}-\frac{1}{2 \kappa_{1}} \ln \left(\frac{z_{d}+\zeta_{1} \pi n / \kappa_{1}+i\left(\zeta_{1} \kappa_{0} / \kappa_{1}-\zeta_{0}\right)}{z_{d}-\zeta_{1} \pi n / \kappa_{1}-i\left(\zeta_{1} \kappa_{0} / \kappa_{1}+\zeta_{0}\right)}\right)+O\left(n^{-2}\right) .
$$

Note that expansion (48) can be obtained from formula (51) in the limit $\left|z_{d}\right| \gg \zeta_{1} \pi n / \kappa_{1}$.

For $\left|z_{d}\right| \sim \zeta_{1} \pi n / \kappa_{1}$ solution (51) breaks down in the vicinities of the points where

$$
z_{d}=-\frac{\zeta_{1} \pi n}{\kappa_{1}}-i\left(\frac{\zeta_{1} \kappa_{0}}{\kappa_{1}}-\zeta_{0}\right) \text { or } z_{d}=\frac{\zeta_{1} \pi n}{\kappa_{1}}+i\left(\frac{\zeta_{1} \kappa_{0}}{\kappa_{1}}+\zeta_{0}\right)
$$

If the value of the specific impedance $z_{d}$ is sufficiently close to any of these points, the logarithmic term on the righthand side of the expression (47) becomes comparable with the first term $\pi n / \kappa_{1}$ and the main order solution is not governed by the hard wall approximation any more. Thus, it must be assumed that $z_{d}$ does not lie in the immediate vicinity of the points (52). The case when the logarithmic term on the right-hand side of the expression (47) dominates over $\pi n / \kappa_{1}$ will be studied in what follows. However, the situation when these terms are of the same order of magnitude was found difficult for analytical treatment and was tackled numerically.

Taking the real and imaginary parts of (51) leads to the formulas

$$
\begin{gathered}
\widetilde{k}_{r}-\widetilde{k}_{r}^{0}=-\frac{1}{4 \kappa_{1}} \ln \left\{\frac{\left(R^{2}+\left(\zeta_{0}-X\right)^{2}-A^{2}-B^{2}\right)^{2}+4\left(B R+A\left(\zeta_{0}-X\right)\right)^{2}}{\left[(R-A)^{2}+\left(B+\zeta_{0}-X\right)^{2}\right]^{2}}\right\}, \\
\tan \widetilde{\psi}=-\frac{2\left(B R+A\left(\zeta_{0}-X\right)\right)}{R^{2}+\left(\zeta_{0}-X\right)^{2}-A^{2}-B^{2}}, \quad \widetilde{\psi}=2 \kappa_{1}\left(\widetilde{k}_{i}-\widetilde{k}_{i}^{0}\right) .
\end{gathered}
$$

Here $A=\zeta_{2} \pi n / \kappa_{1}, B=\zeta_{1} \kappa_{0} / \kappa_{1}$. Assume now that $|A| \sim|X| \gg R$. Relations $(53,54)$ then can be used to write $\widetilde{k}_{r}$ in terms of $\widetilde{k}_{i}$. Indeed,

$$
\left(\zeta_{0}-X\right) \approx \frac{A}{\sin \widetilde{\psi}}(\cos \widetilde{\psi} \pm 1), \quad \widetilde{k}_{r}-\widetilde{k}_{r}^{0} \approx \frac{1}{\kappa_{1}} \frac{B\left(\zeta_{0}-X\right)-R A}{\left(\zeta_{0}-X\right)^{2}+A^{2}}
$$

and hence,

$$
\begin{array}{ll}
\widetilde{k}_{r}-\widetilde{k}_{r}^{0}=\frac{-R \cos \left(2 \kappa_{1}\left(\widetilde{k}_{i}-\widetilde{k}_{i}^{0}\right)\right)+B \sin \left(2 \kappa_{1}\left(\widetilde{k}_{i}-\widetilde{k}_{i}^{0}\right)\right)-R}{2 A \kappa_{1}}, & \text { if } X / A>0, \\
\widetilde{k}_{r}-\widetilde{k}_{r}^{0}=\frac{-R \cos \left(2 \kappa_{1}\left(\widetilde{k}_{i}-\widetilde{k}_{i}^{0}\right)\right)-B \sin \left(2 \kappa_{1}\left(\widetilde{k}_{i}-\widetilde{k}_{i}^{0}\right)\right)+R}{2 A \kappa_{1}}, & \text { if } X / A<0 .
\end{array}
$$

Formulas $(55,56)$ show that when the real part of the specific impedance $R$ is small and kept fixed while the imaginary part of the specific impedance $X$ varies, the eigenvalue $\widetilde{k}$ with a sufficiently large index $n$ must move along the sinusoidal line. The zeros of this line coincide with the hard-wall eigenvalues $\widetilde{k}^{0}=\widetilde{k}_{r}^{0}+\widetilde{k}_{i}^{0}$. For instance, if for some $n>0$ (or $n<0$ ) the correspondent eigenvalue $\widetilde{k}^{0}(n)$ is taken as a starting point at $X=+\infty$ and then $X$ is allowed to gradually vary till $X=-\infty$, then moving along the line (55) (or (56), respectively) the eigenvalue value $\widetilde{k}$ will terminate at the point $\widetilde{k}^{0}(n+1)$ (or $\widetilde{k}^{0}(n-1)$, respectively). This can be also noticed directly from formula (51). Indeed, as the point $z_{d}$ moves along the line parallel to the imaginary axis from the position $z_{d}=R+i \infty$ to $z_{d}=R-i \infty$ and passes by singular points (52), but does not cross them, the imaginary part of the logarithm in (51) increases by $-2 \pi$. As a result, the imaginary part of the eigenvalue value $\widetilde{k}$ gets the overall increment of $\pi / \kappa_{1}$. The amplitude of the sinusoidal lines $(55,56)$ decays as $1 / n$ as $n \rightarrow \infty$. Note that the obtained results are also in line with the related numerical findings presented in figure 7 of Rienstra [16].

\section{Surface modes}

Only small corrections to the hard-wall solutions have been considered so far. The first of such solutions was based on the assumption that $R \gg \zeta_{1} \pi n / \kappa_{1} \gg 1$ and corresponded to the emergence of circular eigenvalue contours (49) in the complex plane $\widetilde{k}$. The second solution was obtained in the limit $\zeta_{1} \pi n / \kappa_{1} \gg R$ and resulted in sinusoidal eigenvalue contours $(55,56)$. Consider now the case when $z_{0}$ is close to either of the points $\pm z_{d}$ and the logarithmic term in 
(46) is large. To construct relevant solutions assume that $|X|$ is large, the wall Mach number $M_{d}$ does not vanish and $\widetilde{k} \rightarrow \infty$. Dispersion relation (46) then can be rewritten in the following form

$$
e^{-2 \kappa_{1}\left(\widetilde{k}+\kappa_{0}+O\left(\widetilde{k}^{-1}\right)\right)}=\frac{z_{d}-i\left(\zeta_{1} \widetilde{k}-\zeta_{0}+O\left(\widetilde{k}^{-1}\right)\right)}{z_{d}+i\left(\zeta_{1} \widetilde{k}-\zeta_{0}+O\left(\widetilde{k}^{-1}\right)\right)}
$$

Its main-order solution is

$$
\widetilde{k}=-i z_{d} / \zeta_{1}+\ldots \quad \text { if } X \rightarrow-\infty \quad \text { and } \widetilde{k}=i z_{d} / \zeta_{1}+\ldots \quad \text { if } X \rightarrow+\infty
$$

These solutions are analogous to the high-frequency surface-wave modes studied in Rienstra [16] in the context of a hollow duct in a uniform flow. It is interesting to observe that although they do not have a hard-wall prototype, the eigenvalues (58) follow from the same dispersion relation as the rest of the acoustic modes. When the wall Mach number $M_{d}=0$, formulas (58) do not apply. This situation has to be tackled numerically.

\section{B. Turning points}

Assume that the function $q(r)$ from (36-38) has a simple zero at a point $r=r_{*}, s<r_{*}<1$. Then, as is known (see for instance Barantsev \& Engelgart [15]), ordinary WKB procedure is not valid near $r_{*}$ and should be modified so as to retain the structure of the function $q(r)$ in the main part of the operator on the right-hand side of (36). This can be accomplished by taking

$$
\tau(r)=\left\{\frac{3}{2} \int_{r_{*}}^{r} \sqrt{q(\xi)} d \xi\right\}^{2 / 3} .
$$

Then assuming that $m \sim \widetilde{\omega} \sim \widetilde{k}$ as $\widetilde{k} \rightarrow \infty$ and (40), the problem (36-38) becomes

$$
\frac{\mathrm{d}^{2} w}{\mathrm{~d} \tau^{2}}+\tau w=0, \quad \frac{\mathrm{d} w}{\mathrm{~d} \tau}+i \sigma_{h} w=0 \quad \text { at } \tau=\tau_{h}, \quad \frac{\mathrm{d} w}{\mathrm{~d} \tau}-i \sigma_{d} w=0, \quad \text { at } \tau=\tau_{d},
$$

here $\tau_{h}=\tau(s), \tau_{d}=\tau(1), \sigma_{h}$ and $\sigma_{d}$ are given by (42). System (59) has solution $w(\tau)=A A i(-\tau)+B B i(-\tau)$ which is not identically zero if

$$
I(\tilde{k})=\operatorname{det}\left\|\begin{array}{ll}
A i^{\prime}\left(-\tau_{d}\right)+i \sigma_{d} A i\left(-\tau_{d}\right) & B i^{\prime}\left(-\tau_{d}\right)+i \sigma_{d} B i\left(-\tau_{d}\right) \\
A i^{\prime}\left(-\tau_{h}\right)-i \sigma_{h} A i\left(-\tau_{h}\right) & B i^{\prime}\left(-\tau_{h}\right)-i \sigma_{h} B i\left(-\tau_{h}\right)
\end{array}\right\|=0 .
$$

For the sake of simplicity we shall consider only the case when the inner wall is hard so that $\sigma_{h}=0$ in (59) and (60).

To main order in $\widetilde{k}$ the position of the turning point is given by the equation $\left(\widetilde{\omega}-\widetilde{k} M\left(r_{*}\right)\right)^{2}-\widetilde{k}^{2}-m^{2} / r_{*}^{2}=0$, or equivalently,

$$
\widetilde{k}=\frac{-\widetilde{\omega} M\left(r_{*}\right) \pm \sqrt{\widetilde{\omega}^{2}-\left(1-M^{2}\left(r_{*}\right)\right) m^{2} / r_{*}^{2}}}{\left(1-M^{2}\left(r_{*}\right)\right)} .
$$

Since $r_{*}>s>0$, assume in what follows that the non-dimensional frequency $\widetilde{\omega}$ is large enough for the square root in the above formula to be a real number. This is also the most practically important situation. In this case the lateral wavenumber $\widetilde{k}$ and the function $q(r)$ are also real and, hence, either $q^{\prime}\left(r_{*}\right)>0$ or $q^{\prime}\left(r_{*}\right)<0$. For determinacy consider the first inequality. Then as $\widetilde{k} \rightarrow \infty$

$$
\tau_{h}=\left\{-\frac{3}{2} i \int_{s}^{r_{*}} \sqrt{|q(\xi)|} \mathrm{d} \xi\right\}^{2 / 3} \rightarrow-\infty, \quad \tau_{d}=\left\{\frac{3}{2} \int_{r_{*}}^{1} \sqrt{q(\xi)} \mathrm{d} \xi\right\}^{2 / 3} \rightarrow+\infty
$$

and the dispersion relation (60) yields

$$
\cos \left(\zeta_{d}+\frac{1}{4} \pi\right)-i \sigma_{d} \tau_{d}^{-1 / 2} \sin \left(\zeta_{d}+\frac{1}{4} \pi\right)+\ldots=0
$$

where $\zeta_{d}=\int_{r_{*}}^{1} \sqrt{q(\xi)} \mathrm{d} \xi$. Substituting $\sigma_{d}$ from (42), $z_{d}=R+i X$, using the identity $\tau^{\prime} \tau^{1 / 2}=\sqrt{q(r)}$ and taking the real and imaginary part of (61) we obtain the system

$$
\left\{\begin{aligned}
\cos \left(\zeta_{d}+\frac{1}{4} \pi\right)-\frac{X}{R^{2}+X^{2}} \frac{\left(\widetilde{\omega}-\tilde{k} M_{d}\right)^{2}}{\widetilde{\omega} \sqrt{q(1)}} \sin \left(\zeta_{d}+\frac{1}{4} \pi\right) & =0 \\
\frac{R}{R^{2}+X^{2}} \sin \left(\zeta_{d}+\frac{1}{4} \pi\right) & =0 .
\end{aligned}\right.
$$


If $z_{d}=\infty$, (62) reduces to the hard-wall case considered in Cooper and Peake [9] (see their formula (103) with $\psi=0$ )

$$
\int_{r_{*}}^{1} \sqrt{q(r)} \mathrm{d} r=\pi\left(n+\frac{1}{4}\right), \quad n=0,1,2,3 \ldots
$$

It follows from (62) that the problem with lined walls does not admit real turning points. The exception is a very special case of $R=0$ when system (62) reduces to the equation

$$
\sqrt{\left(\widetilde{\omega}-\widetilde{k} M_{d}\right)^{2}-\widetilde{k}^{2}-m^{2}}=\frac{\left(\widetilde{\omega}-\widetilde{k} M_{d}\right)^{2}}{\widetilde{\omega} X} \tan \left(\zeta_{d}+\frac{1}{4} \pi\right) .
$$

As with the ordinary WKB approximation, it is useful to check the accuracy of the approximate formula (63) against the related exact uniform flow solutions with $\widetilde{k}$ real. The results of the comparison are shown in Table 2 for the same values of governing parameters as in Tables 1: $\widetilde{\omega}=25, m=15, s=0.6, M=0.5$. In computation of the relative error in Table 2 the adjustment for the Doppler shift $\widetilde{k}_{\mathrm{d}}$ in $\widetilde{k}$ equal to $-16.6(6)$ was made. It can be seen from Table 2 that the accuracy is reasonably good, although it decreases significantly when the turning point is located very close to the duct wall. This situation is illustrated in line 4 of the table. Here the turning point actually lies slightly inside the hub, i.e., $r_{t . p .}=0.589<s=0.6$ and the related eigenvalue must be taken from the ordinary WKB analysis. However, the turning point prediction was found to be in perceptibly better agreement with the exact solution.

Table 2. Turning point analysis versus exact solution

\begin{tabular}{cccc} 
No & WKB TP & Exact & Error \% \\
& $\widetilde{k}_{\mathrm{tp}}$ & $\widetilde{k}$ & $\left|\widetilde{k}_{\mathrm{tp}}-\widetilde{k}\right| /\left|\widetilde{k}-\widetilde{k}_{\mathrm{d}}\right|$ \\
\hline 1 & -43.36 & -43.59 & 1.38 \\
2 & -38.00 & -38.36 & 2.16 \\
3 & -31.12 & -32.08 & 6.23 \\
4 & -2.210 & -1.251 & 6.22 \\
5 & 4.564 & 5.028 & 2.14 \\
6 & 10.45 & 10.26 & 0.72
\end{tabular}

\section{Hydrodynamic spectrum}

The analysis carried out in the previous sections assumed that the quantity $\widetilde{\omega}-\widetilde{k} M(r)$ did not vanish, so that the coefficients of the governing equation remained smooth. This section aims to study the properties of the eigensolutions of system (32-34) when $\widetilde{\omega}-\widetilde{k} M(r)=0$ at some point $r=\widetilde{r}, s \leq \widetilde{r} \leq 1$. In this case the coefficient before the first derivative in equation (32) goes to infinity and the function $q(r)$ defined by (39) develops a singularity near $\tilde{r}$. As a result, ordinary WKB solution constructed above becomes invalid at the point $\widetilde{r}$, although it still gives the correct outer limit of the solution for large $\widetilde{k}$ as $r \rightarrow \widetilde{r}$. In order to clarify the structure of the inner solution near $\widetilde{r}$, at first we consider a model problem for a slender duct. The procedure of matching of the outer WKB solution with the corresponding inner solution which does not assume that the duct is slender is described in the second part of this section. This procedure can be extended to a more general equation (25), in order to include the effects of the meanflow swirl. It should be noted, however, that equation (25) has the stronger singularity than equation (32) and the results of this section are not directly applicable to the swirling flow. For the sake of simplicity in this section only the case of hard-walls will be considered.

\section{Slender duct}

Let $\Delta_{+}=1-\widetilde{r}, \Delta_{-}=\widetilde{r}-s, \Delta=1-s$, where $0<s \leq \widetilde{r} \leq 1$, and assume that $\Delta \ll 1$. Introduce new independent variable $\eta=(r-\widetilde{r}) / \Delta$. Note that $\eta=O(1)$ since $-\Delta_{-} / \Delta \leq \eta \leq \Delta_{+} / \Delta$. Then

$$
r^{-1}=\widetilde{r}^{-1}+O(\Delta), \quad \widetilde{\omega}-\widetilde{k} M(r)=-\Delta \widetilde{k}\left(M^{\prime}(\widetilde{r}) \eta+O(\Delta)\right), \quad M(r)=M^{\prime}(\widetilde{r})+O(\Delta)
$$

and equation (32) becomes, to leading order in $\Delta$,

$$
\frac{\mathrm{d}^{2} P}{\mathrm{~d} \eta^{2}}-\frac{2}{\eta} \frac{\mathrm{d} P}{\mathrm{~d} \eta}-v^{2} P=0, \quad \frac{\mathrm{d} P}{\mathrm{~d} \eta}\left(\eta=-\frac{\Delta_{-}}{\Delta}\right)=\frac{\mathrm{d} P}{\mathrm{~d} \eta}\left(\eta=\frac{\Delta_{+}}{\Delta}\right)=0,
$$


here $v^{2}=\Delta^{2}\left(\widetilde{k}^{2}+m^{2} / \widetilde{r}^{2}\right)$. The first equation of system (65) has general solution

$$
P=c_{+}(1-v \eta) \exp (v \eta)+c_{-}(1+v \eta) \exp (-v \eta)
$$

with $c_{-}, c_{+}=$const. Substitution of (66) into the boundary conditions leads to the following system for $c_{-}$and $c_{+}$

$$
\begin{gathered}
\frac{\mathrm{d} P}{\mathrm{~d} \eta}\left(\eta=-\frac{\Delta_{-}}{\Delta}\right)=v^{2} \frac{\Delta_{-}}{\Delta}\left(c_{+} \exp \left(-v \frac{\Delta_{-}}{\Delta}\right)+c_{-} \exp \left(v \frac{\Delta_{-}}{\Delta}\right)\right)=0, \\
\frac{\mathrm{d} P}{\mathrm{~d} \eta}\left(\eta=\frac{\Delta_{+}}{\Delta}\right)=-v^{2} \frac{\Delta_{+}}{\Delta}\left(c_{+} \exp \left(v \frac{\Delta_{+}}{\Delta}\right)+c_{-} \exp \left(-v \frac{\Delta_{+}}{\Delta}\right)\right)=0 .
\end{gathered}
$$

This system does not permit non-zero solutions for real $\tilde{k}$ unless either $\Delta_{+}=0$ or $\Delta_{-}=0$. Each of these cases leads to the eigensolution

$$
P=(1+\nu \eta) \exp (-v(\eta-1))-(1-v \eta) \exp (\nu(\eta-1))
$$

where $\eta=(r-1) / \Delta, v=\Delta \sqrt{\tilde{k}^{2}+m^{2}}, \widetilde{k}=\widetilde{\omega} / M_{d}, \quad M_{d} \neq 0$ for $\tilde{r}=1$ and $\eta=(r-s) / \Delta, v=\Delta \sqrt{k^{2}+m^{2} / s^{2}}$, $\widetilde{k}=\widetilde{\omega} / M_{h}, M_{h} \neq 0$ for $\widetilde{r}=s$.

\section{General case}

For large $\widetilde{k}$ and away from the point $r=\widetilde{r}$ the WKB-solution to the Pridmore-Brown equation (32) which satisfies the hard-wall boundary conditions is

$$
P=\frac{\widetilde{\omega}-\widetilde{k} M}{\sqrt{r}}\left(\tau^{\prime}\right)^{-1 / 2} \cos (\tau)+\ldots, \quad \tau(r)=\int_{a}^{r} \sqrt{q(\xi)} \mathrm{d} \xi,
$$

where $a=s>0$, if $s \leq r<\widetilde{r}$ and $a=1$, if $\widetilde{r}<r \leq 1$. If $r$ is kept fixed and such that $r \neq \widetilde{r}$ and $\widetilde{k} \rightarrow \infty$ the function $q(r)$ previously defined by the expression (39) becomes, to leading order,

$$
q(r)=q_{0}(r), \quad \text { where } q_{0}(r)=(\widetilde{\omega}-\widetilde{k} M)^{2}-\widetilde{k}^{2}-m^{2} / r^{2},
$$

since for the most singular term in (39) holds the estimate

$$
\frac{2 \widetilde{k}^{2}\left(M^{\prime}(r)\right)^{2}}{(\widetilde{\omega}-\widetilde{k} M(r))^{2}}=\frac{2\left(M^{\prime}(r)\right)^{2}}{(M(\widetilde{r})-M(r))^{2}} \leq \frac{\mathrm{const}}{(\widetilde{r}-r)^{2}} \ll \widetilde{k}^{2},
$$

provided $|\widetilde{r}-r| \gg 1 / \widetilde{k}$ and $M^{\prime}(r)$ is bounded for $s \leq r \leq 1$. In order to obtain outer asymptotic expansion of the solution near the point $\widetilde{r}$, substitute (69) in (68) and take the limit $r \rightarrow \widetilde{r} \pm$. This gives

$$
\begin{aligned}
& P=-\frac{\widetilde{k} M^{\prime}(\widetilde{r})(r-\widetilde{r})}{2 \sqrt{r \mu i}}\left(e^{-I_{-}-\mu(r-\widetilde{r})}+e^{I_{-}+\mu(r-\widetilde{r})}\right)+\ldots \quad \text { as } r \rightarrow \widetilde{r}-, \\
& P=-\frac{\widetilde{k} M^{\prime}(\widetilde{r})(r-\widetilde{r})}{2 \sqrt{r \mu i}}\left(e^{-I_{+}-\mu(r-\widetilde{r})}+e^{I_{+}+\mu(r-\widetilde{r})}\right)+\ldots \text { as } r \rightarrow \widetilde{r}+,
\end{aligned}
$$

where

$$
I_{-}=\int_{s}^{\widetilde{r}} \sqrt{-q_{0}(r)} \mathrm{d} r, \quad I_{+}=\int_{1}^{\widetilde{r}} \sqrt{-q_{0}(r)} \mathrm{d} r, \quad \mu=\sqrt{\widetilde{k}^{2}+m^{2} / \widetilde{r}^{2}} .
$$

Owing to the singularity $(70)$, formulas $(71,72)$ are invalid in the region, where $|\widetilde{r}-r| \sim 1 / \widetilde{k}$, since in this region the singularity becomes comparable with the leading order term $-\widetilde{k}^{2}-m^{2} / r^{2}$ in the expansion of $q(r)$. Prompted by the solution (67) of the model problem, introduce local coordinate $\xi=\mu(r-\widetilde{r})$ which is centred on the singular point $\tilde{r}$ and is $O(1)$ in the region where $|\widetilde{r}-r| \sim 1 / \widetilde{k}$. Then, to leading order, the Pridmore-Brown equation becomes

$$
\frac{\mathrm{d}^{2} P}{\mathrm{~d} \xi^{2}}-\frac{2}{\xi} \frac{\mathrm{d} P}{\mathrm{~d} \xi}-P=0 .
$$

It must be supplemented with the conditions of matching with the main-order solution $(71,72)$ obtained in the outer region, i.e.,

$$
P(\xi \rightarrow \pm \infty) \rightarrow A \xi\left(e^{-\left(I_{ \pm}+\xi\right)}+e^{\left(I_{ \pm}+\xi\right)}\right)+\ldots, \quad A=-\frac{\widetilde{k} M^{\prime}(\widetilde{r})}{2 \sqrt{\widetilde{r} \mu^{3} i}}
$$


Equation (73) has general solution

$$
P=c_{+}(1-\xi) \exp (\xi)+c_{-}(1+\xi) \exp (-\xi) .
$$

It can satisfy the restrictions at the infinity only provided $c_{+}=-A \exp \left(I_{+}\right), c_{-}=A \exp \left(I_{-}\right)$and $I_{+}-I_{-}=2 \pi i n$, where $n$ is an integer. The latter restriction can be rewritten as

$$
\int_{s}^{1} \sqrt{\widetilde{k}^{2}+m^{2} / r^{2}-(\widetilde{\omega}-\widetilde{k} M)^{2}} \mathrm{~d} r=2 \pi i n
$$

where $\widetilde{\omega} / \max (M(r)) \leq \widetilde{k} \leq \widetilde{\omega} / \min (M(r))$ for $s<r<1$. Substitution of $\widetilde{\omega}=\widetilde{k} M(\widetilde{r})$ into equation (76) gives

$$
\int_{s}^{1} \sqrt{\left[1-(M(\widetilde{r})-M(r))^{2}\right] \widetilde{k}^{2}+m^{2} / r^{2}} \mathrm{~d} r=2 \pi i n .
$$

which is impossible for a subsonic mean flow. Thus, the only possibility is that $\widetilde{r}=s$ or $\widetilde{r}=1$. In the first case (74) gives way to the following boundary conditions

$$
P(\xi \rightarrow+\infty) \rightarrow A \xi\left(e^{-\left(I_{+}+\xi\right)}+e^{\left(I_{+}+\xi\right)}\right)+\ldots, \quad \frac{\mathrm{d} P}{\mathrm{~d} \xi}(0)=0
$$

so that $c_{+}=-A \exp \left(I_{+}\right), c_{-}=A \exp \left(-I_{+}\right), \widetilde{k}=\widetilde{\omega} / M_{h}$ in the solution (75). In the second case the appropriate boundary conditions are

$$
P(\xi \rightarrow-\infty) \rightarrow A \xi\left(e^{-\left(I_{-}+\xi\right)}+e^{\left(I_{-}+\xi\right)}\right)+\ldots, \quad \frac{\mathrm{d} P}{\mathrm{~d} \xi}(0)=0,
$$

and $c_{+}=-A \exp \left(I_{-}\right), c_{-}=A \exp \left(-I_{-}\right), \widetilde{k}=\widetilde{\omega} / M_{d}$, respectively.

Overall, the results of this section show that if the structure of the boundary-layer flow in the immediate vicinity of the duct walls is discarded and the lateral component of the mean flow velocity is assumed non-zero on the walls, then for sufficiently large $\widetilde{k}$ hydrodynamic modes of Pridmore-Brown equation are localized near the walls and their phase velocity is close to the mean flow velocity at the walls. As it will be clear from what follows, this may be not true, however, if the mean flow velocity has typical boundary-layer flow profile that satisfies no-slip conditions on the walls.

It is useful to compare the results of this section to the multiple-scale analysis of Golubev \& Atassi [6] obtained for the mean flow with the solid-body swirl. The above authors demonstrate the existence of a critical layer centred on the purely convective lateral wavenumber, where the accumulation of nearly convective eigenvalues takes place for non-zero swirl. Remarkably enough, if the swirl is set to zero in their dispersion relation (43), the nearly-convected modes disappear and the only remaining eigenvalue is the purely convective mode which is also the case here. Note that clustering of nearly-convective modes was reported in Golubev \& Atassi [6] for a differential equation which does not reduce to the Pridmore-Brown equation studied in this section.

\section{Numerical procedure}

The eigenvalue system $(25,27,28)$ was solved numerically using the following method. The field equation $(25)$ was first rewritten in the form of a system of two first-order ordinary differential equations

$$
u=P^{\prime}, \quad u^{\prime}=-\beta(r) u-\gamma(r) P,
$$

where $\beta(r)$ and $\gamma(r)$ are the coefficients before $P^{\prime}$ and $P$ in equation (25). For zero circumferential mean flow velocity they are identical to $\beta(r)$ and $\gamma(r)$ appearing in the Pridmore-Brown equation (29). To reduce the amount of computations these coefficients were rewritten as follows

$$
\beta(r)=\frac{B}{\lambda}+\Pi+\frac{1}{\lambda} \frac{\mathrm{d}}{\mathrm{d} r}(\lambda), \quad \gamma(r)=\frac{A \Omega}{\lambda^{2}}+\frac{1}{\lambda} \frac{\mathrm{d}}{\mathrm{d} r}(B)+\frac{B \Pi}{\lambda}, \quad \Pi=\frac{1}{r}-\frac{1}{\bar{\rho} A} \frac{\mathrm{d}}{\mathrm{d} r}(\bar{\rho} A)+C,
$$

so that their common part $\Pi$ could be computed only once per run. System (78) was approximated by the following implicit finite-difference scheme

$$
\frac{P^{j+1}-P^{j}}{\delta}=u^{j+1}, \quad \frac{u^{j+1}-u^{j}}{\delta}=-\beta^{j+1} u^{j+1}-\gamma^{j+1} P^{j+1}, \quad j=0,1,2,3, \ldots N-1
$$


where $\delta=(d-h) / N, r^{j}=h+j \delta, j=0,1,2,3, \ldots N, N$ is a sufficiently large integer number. Since system (79) is linear, it was solved with respect to the unknown variables $u^{j+1}$ and $P^{j+1}$

$$
P^{j+1}=\frac{\left(1+\delta \beta^{j+1}\right) P^{j}+\delta u^{j}}{1+\delta \beta^{j+1}+\delta^{2} \gamma^{j+1}}, \quad u^{j+1}=\frac{P^{j+1}-P^{j}}{\delta}, \quad j=0,1,2,3, \ldots N-1 .
$$

Formulas (80) were supplemented with the initial conditions at $r=r^{0}=h$

$$
P^{0}=1, \quad u^{0}=-\left(\frac{B}{\lambda}+\frac{i \bar{\rho} A}{\omega Z_{h}}\right) P^{0}
$$

and were used together with (81) to compute for a given initial guess of the spectral parameter $k=k_{n}$ the functions $u^{j+1}$ and $P^{j+1}$ and the value

$$
I\left(k_{n}\right)=\frac{1}{M}\left[u^{N}+\left(\frac{B}{\lambda}-\frac{i \bar{\rho} A}{\omega Z_{d}}\right) P^{N}\right], \quad M=\int_{h}^{d}|P| \mathrm{d} r .
$$

Here $I\left(k_{n}\right)$ measures the error in the boundary condition on the wall $r=r^{N}=d$, the role of the parameter $M$ is equivalent to renormalisation of the solution after each run in $r$. Generally, it was not necessary unless very high frequencies $\omega$ and circumferential wavenumbers $m$ were used (say, for Helmholtz number equal to 80 and $m=50$ ). Since normally $I\left(k_{n}\right) \neq 0$ for an arbitrary guess value $k_{n}$, global Newtonian iterations in $k$ were needed to insure the equality $I(k)=0$. The following formula

$$
k_{n+1}=k_{n}-\frac{I\left(k_{n}\right)}{\frac{\mathrm{d} I\left(k_{n}\right)}{\mathrm{d} k}}
$$

was used to update the value of the spectral parameter $k$. The amount of computations in (83) could be reduced in comparison with the classical Newton's method if the derivative $\mathrm{d} I\left(k_{n}\right) / \mathrm{d} k$ was approximated by the finite difference $\left(I\left(k_{n}\right)-I\left(k_{n-1}\right)\right) /\left(k_{n}-k_{n-1}\right)$ (modified Newton's method). The iteration process was continued until the difference between the two successive values of $I\left(k_{n}\right)$ became smaller than a given threshold value.

Table 3. WKB-approximation versus numerical solution

\begin{tabular}{crr}
$\tilde{k}(\mathrm{WKB})$ & $\tilde{k}$ (numerics) & \multicolumn{1}{c}{ error $\%$} \\
\hline$-49.23+i 23.60$ & $-49.17+i 23.78$ & $-0.06-i 0.18$ \\
$-49.34+i 46.67$ & $-49.32+i 46.71$ & $-0.02-i 0.04$ \\
$-49.41+i 62.96$ & $-49.41+i 62.96$ & $0.00-i 0.00$ \\
$-49.46+i 76.88$ & $-49.47+i 76.87$ & $0.01-i 0.01$ \\
$-49.50+i 89.53$ & $-49.52+i 89.51$ & $0.02+i 0.02$
\end{tabular}

An alternative approach with the initial conditions posed on the outer wall $r=r^{N}=d$, formulas (80) employed in the reverse order in $j$ and the iteration procedure organized in order to satisfy the boundary condition on the inner wall $r=r^{0}=h$ was also used. Although it did not have any effect on the acoustic part of the spectrum, in the case of Pridmore-Brown equation it was found more advantageous to march from the inner wall towards the outer wall when computing the hydrodynamic mode localised near the outer wall and to reverse the direction of marching for the hydrodynamic mode localised near the inner wall.

Other numerical schemes with the higher order of approximation than the first-order scheme proposed here were used to solve system (78). Among them were implicit Runge-Kutta, Crank-Nicolson and several other schemes specifically designed for stiff equations (see for details Fornberg \& Driscoll [17]). However, for sufficiently large $m$ and $\omega$ when the stiffness of the system (78) became the most important issue the present scheme was found to be more computationally stable. Our experience with other schemes showed that for large $m$ and $\omega$ there could be difficulties in prediction of the eigensolution with the largest cut-on wavenumber due to the development of numerical instability.

Comparison of numerical results with the exact solutions for the case of uniform mean flow showed numerical solutions to be accurate at least up to four - five decimal digits for the first several hundred eigenvalues with $N \approx$ $1000-3000$.

In the range of large Helmholtz numbers $\widetilde{\omega}$ and $m$ and for non-uniform mean flow the solution was tested against the WKB approximation. An example of such comparison for $\widetilde{\omega}=85, m=45, s=h / d=0.6$ and the mean flow 
Mach number distribution given by the parabola

$$
M=M_{\max } \frac{1+A(\widetilde{r}-s)(1-\widetilde{r})}{1+A(1-s)^{2} / 4}
$$

with $M_{\max }=0.5$ and $A=8$ is presented in Table 3 which shows several first cut-off wavenumbers. The agreement between the WKB predictions of the cut-off modes and their computed values was surprisingly good. For cut-on modes the situation closely mimicked that of Table 2 . The maximum error was about $6 \%$ for the wavenumber with the smallest absolute value and rapidly decreased with increasing $\widetilde{k}$ (not shown).

\section{Numerical results}

\section{A. Ducts with hard walls}

Typical plots of acoustic eigenvalues for three parabolic mean-flow velocity profiles (84) with different extent of nonuniformity $A=1,8,25$ are presented in Figure 1. Also shown is the uniform-flow spectrum. These plots correspond to $\widetilde{\omega}=25, m=15, s=h / d=0.6$ and $M_{\max }=0.5$. Qualitatively all the plots are similar to the uniform-flow case. The only differences are the slight deviation of the first several complex eigenvalues from the vertical direction and the rightward shift of the whole pattern for non-zero $A$ which is due to the decrease of the average speed of the mean flow.

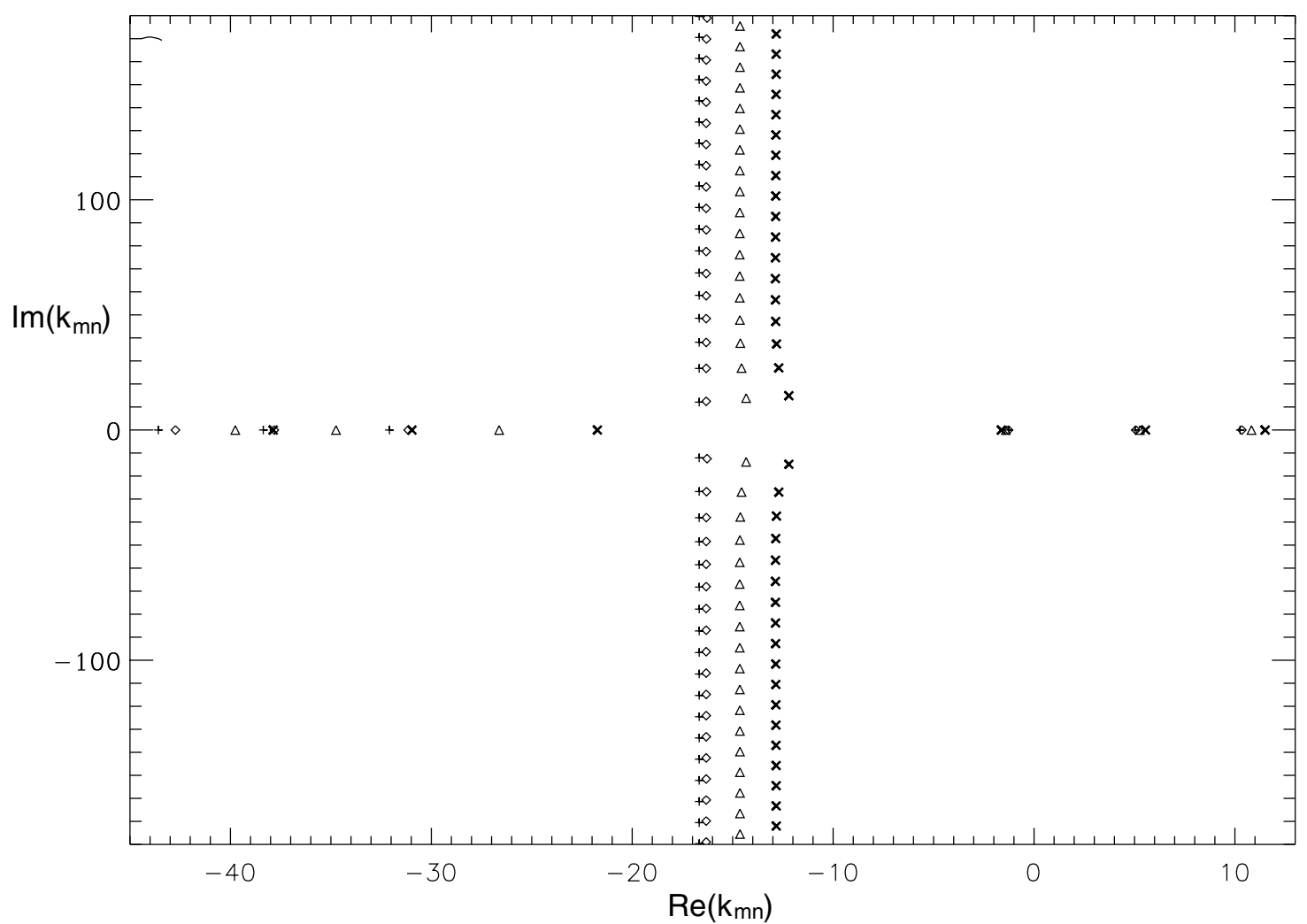

Figure 1. Effect of mean flow non-uniformity (84) on the acoustic part of lateral wavenumber spectrum, $\widetilde{\omega}=25, m=15, s=h / d=0.6$ and $M_{\max }=0.5$. + - uniform flow, $\diamond-A=1\left(M_{d}=0.381\right), \Delta-A=8\left(M_{d}=0.379\right), \times-A=25\left(M_{d}=0.25\right)$.

Computation of the hydrodynamic part of the spectrum for the above example proves to be more difficult. If the number of mesh points $N$ is low (say, $N<3000$ ), several real eigenvalues clustering closely to each other can be observed. However, as the mesh becomes more refined the number of these pseudo-eigenvalues rapidly decreases until only one eigenvalue is left, say $\widetilde{k}=25 / 0.379 \approx 66$ for $A=8$. In agreement with the asymptotic theory proposed above, this eigenvalue corresponds to the Mach number at the duct walls $M_{h}=M_{d} \approx 0.379$. Two eigenfunctions correspond to this eigenvalue. One of them is localized near the inner wall $\widetilde{r}=0.6$ and the other one - near the outer wall $\tilde{r}=1$. They are plotted in Figure 2. Note, that, since the field equation is not symmetric with respect to the mid-radius $\tilde{r}=0.8$, the eigenfunctions shown in Figure 2 are also not symmetric about this point. 


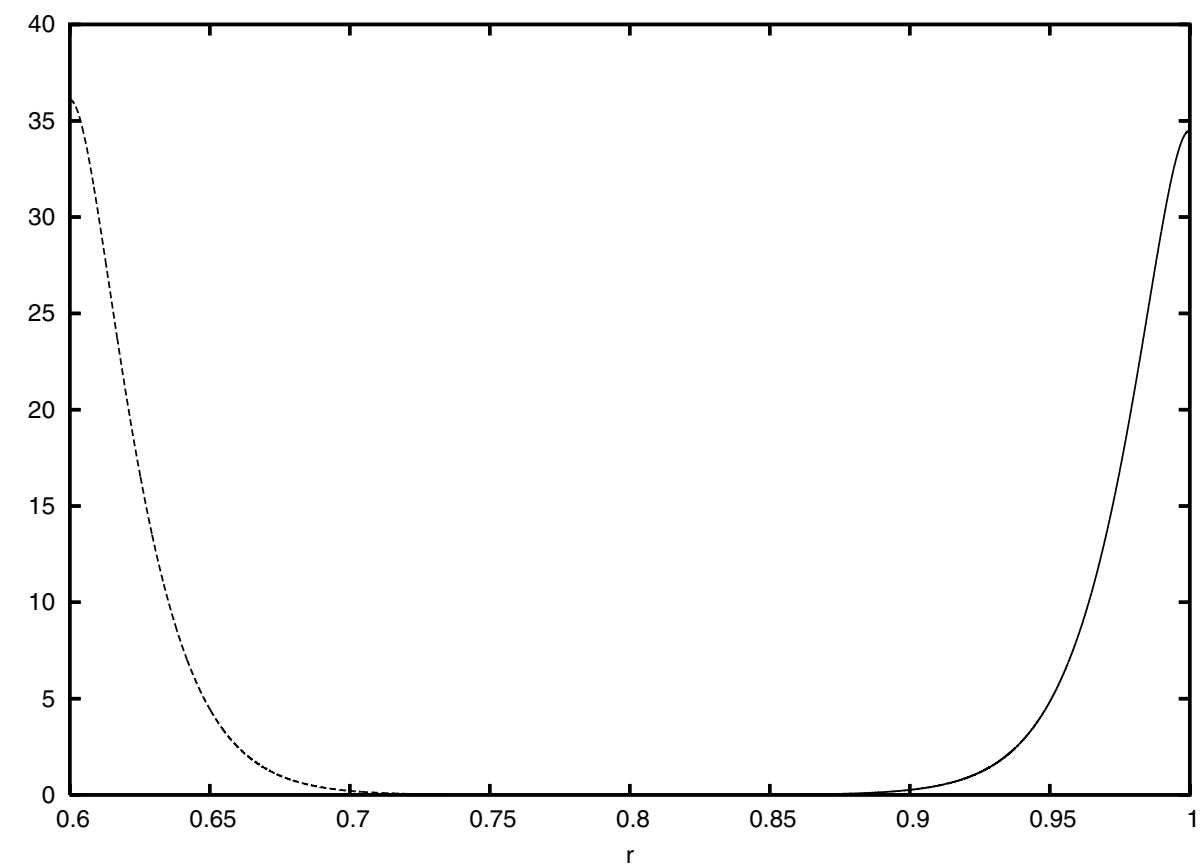

Figure 2. Two hydrodynamic pressure eigenfunctions corresponding to $\widetilde{k}=66, \widetilde{\omega}=25, m=15, s=h / d=0.6$ and $M_{\max }=0.5, A=8$. Each eigenfunction is localized near the corresponding duct wall.

If the wall Mach number is gradually decreased with the maximum Mach number being fixed, these hydrodynamic eigenfunctions become more compactly localised near the duct walls. This situation is illustrated in Figure 3, where two eigenmodes each one corresponding to the parabolic mean-flow velocity profile (84) with $A=8$, and 100 are shown. These values of the parameter $A$ correspond to the inner wall Mach numbers $M_{h} \approx 0.379$ and 0.1 , respectively. For a mean-flow profile without near-wall non-uniformities of the boundary-layer type no new hydrodynamic modes

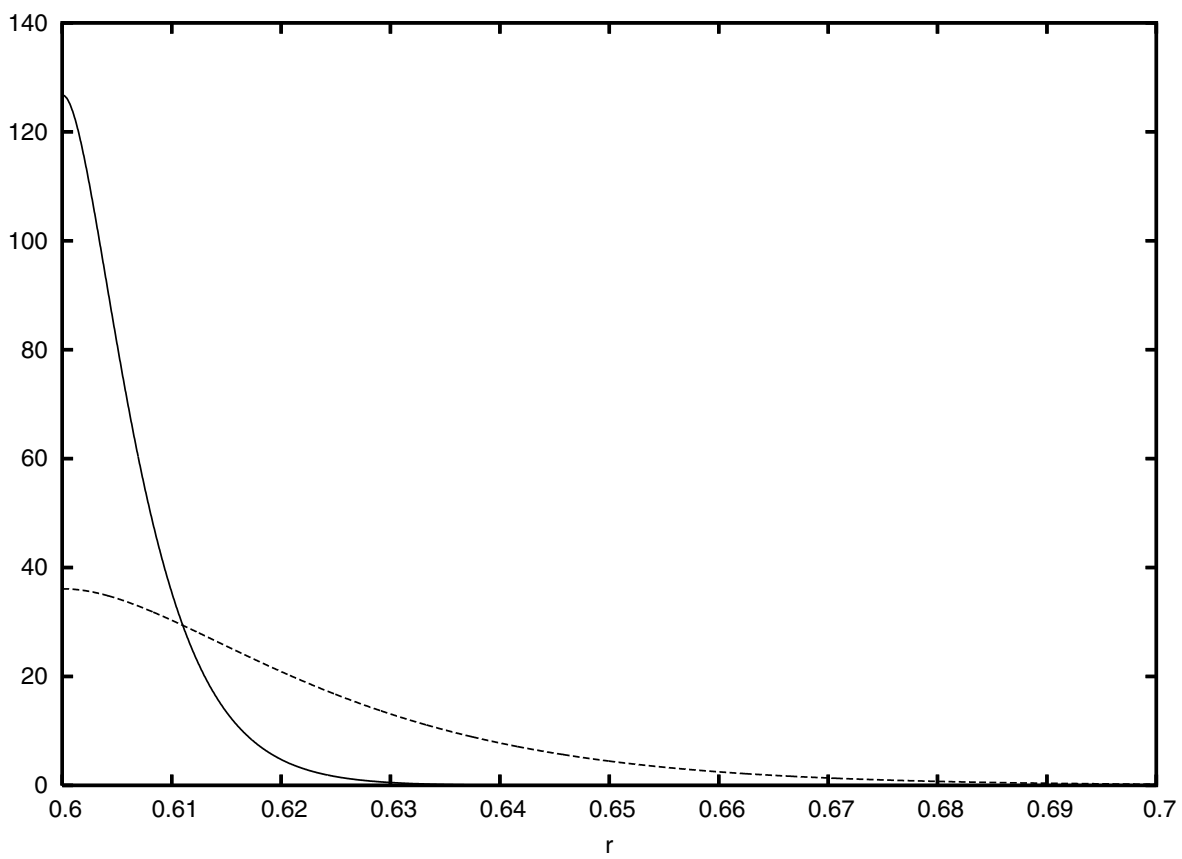

Figure 3. Hydrodynamic pressure eigenfunctions near the inner wall: $\widetilde{\omega}=25, m=15, s=h / d=0.6, M_{\max }=0.5$ and the inner wall Mach numbers $M_{h} \approx 0.379$ and $0.1\left(A=8\right.$, and 100 in (84), respectively). Modes with larger amplitudes correspond to smaller $M_{h}$. 
were found numerically even for very small $M_{h}$.

\section{B. Soft-wall solutions}

This section aims to study the impact of the near-wall shear-layer gradient on behaviour of modal solutions. Hollow lined duct is considered. The following boundary-layer type mean-flow velocity profile is taken

$$
M=M_{\max } \frac{e^{a(1-\widetilde{r})}-e^{-a(1-\widetilde{r})}}{e^{a(1-\widetilde{r})}+e^{-a(1-\widetilde{r})}} .
$$

The parameter $a>0$ controls the steepness of the mean velocity profile near the duct wall $\widetilde{r}=1$. Apart from the limiting case of $a=+\infty$ which corresponds the uniform mean flow, three flow situations with $a=500,250$, and 50 are considered. They correspond to the near-wall "boundary layer thickness" $\delta$ of approximately $1 \%, 1.5 \%$ and $7 \%$ of the total duct radius, with the rest of the mean flow being virtually uniform. As in Rienstra [16], maximum Mach number $M_{\max }=0.5$, Helmholtz number $\widetilde{\omega}=5$ and $m=1$ are used throughout this section.

Figure 4 shows typical trajectories of the lateral wavenumber $\widetilde{k}$ when the imaginary part $X$ of the specific impedance $z_{d}$ varies from minus to plus infinity while its real part remains fixed and relatively large $(R=2)$. The case of the

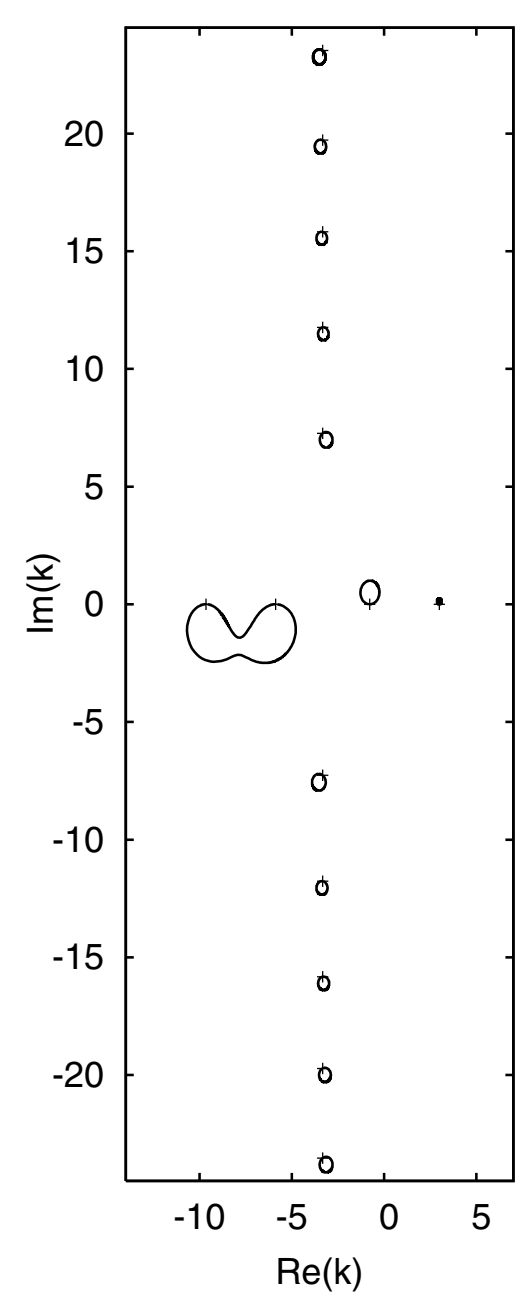

(a) uniform flow

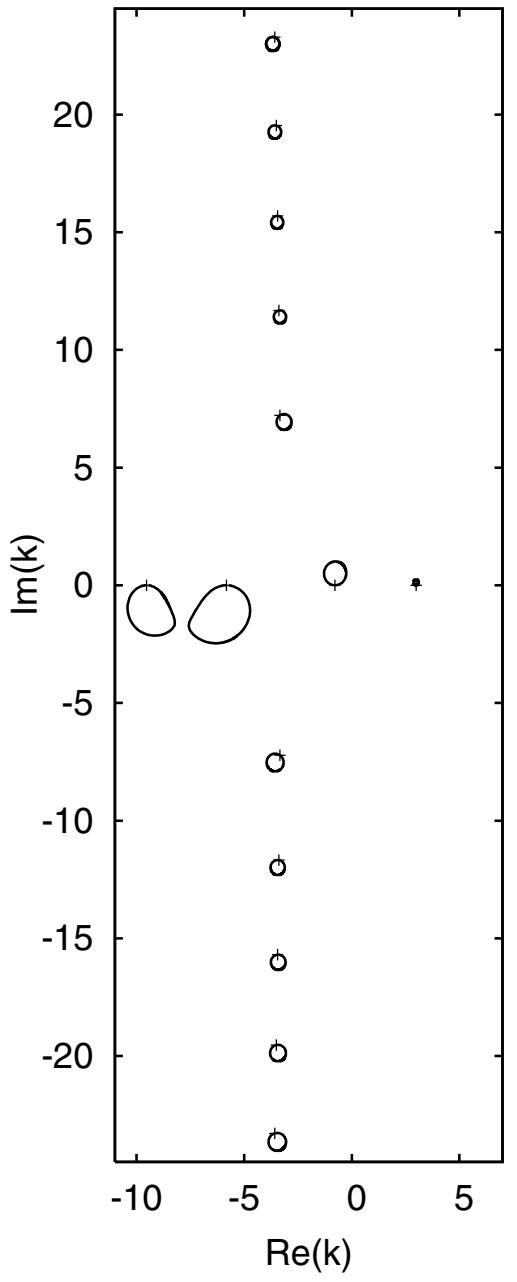

(b) mean-flow profile $a=250$

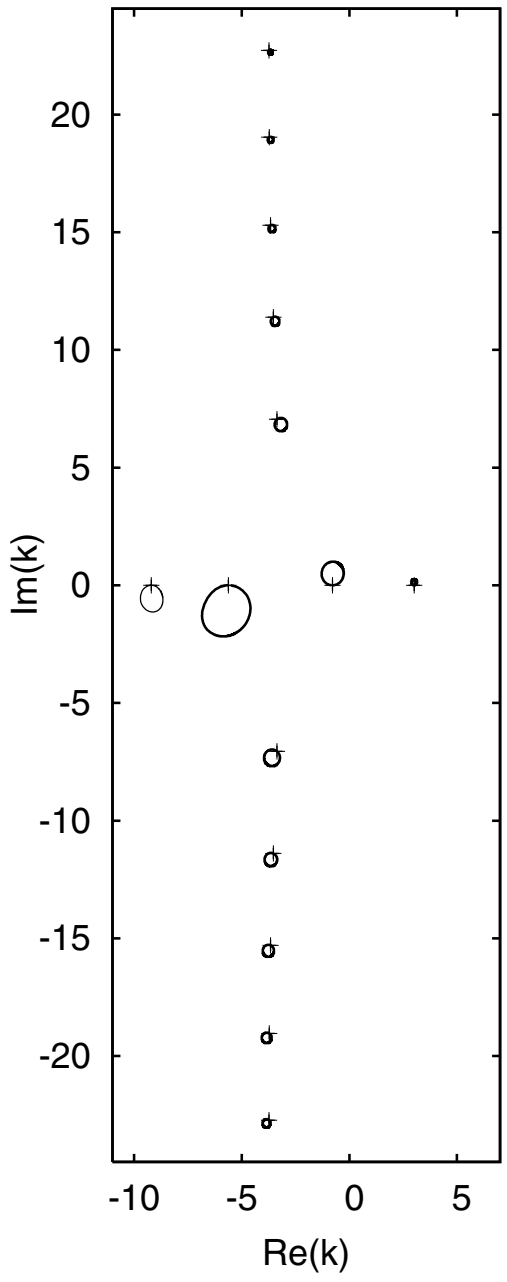

(c) mean-flow profile $a=50$

Figure 4. Trajectories of $\tilde{k}$ for $\widetilde{\omega}=5, m=1, M_{\max }=0.5, Z_{d}=2+i X,-\infty<X<\infty$ : (a) - uniform mean flow, (b) - mean-flow velocity profile (85) with $a=250(\delta \approx 0.015 d)$, (c) - mean-flow velocity profile (85) with $a=50(\delta \approx 0.07 d)$. The hard wall eigenvalues are marked with the "+" sign. 


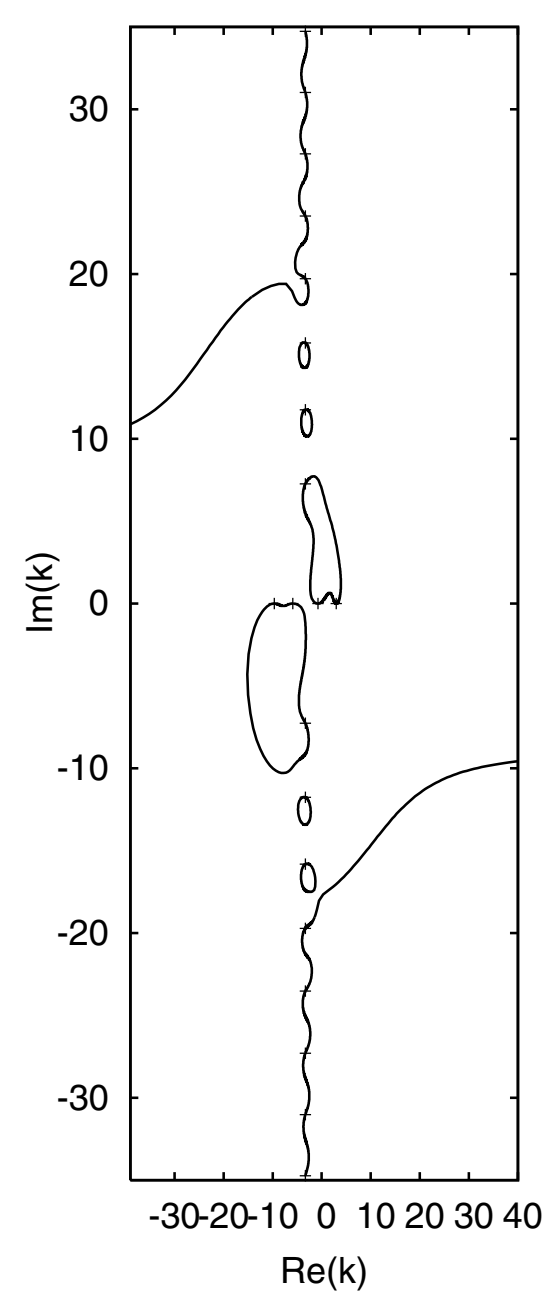

(a) uniform flow

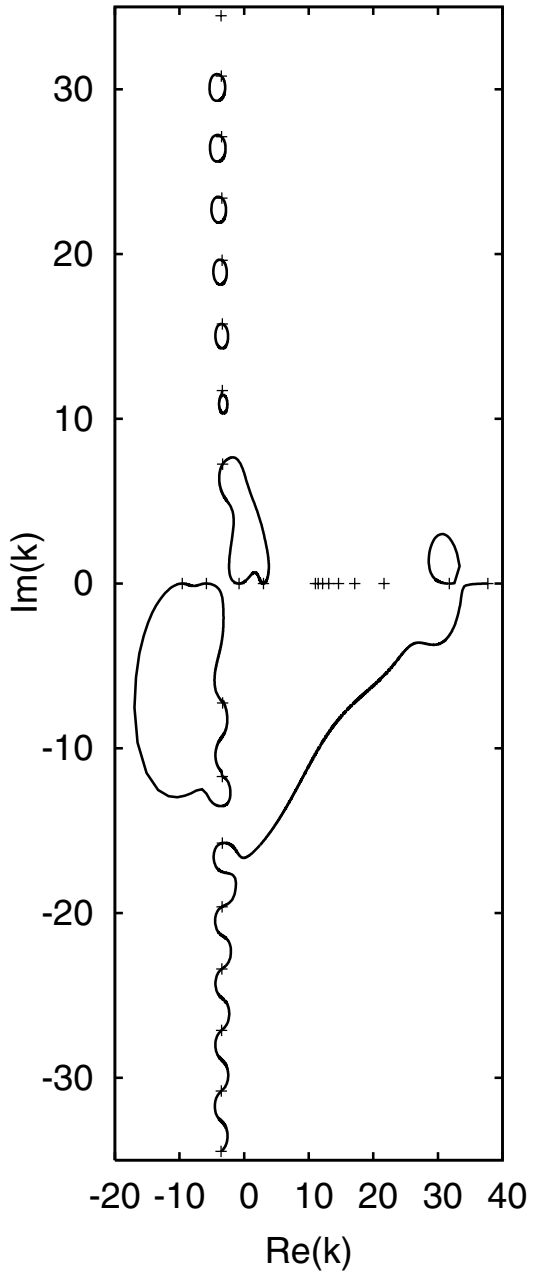

(b) mean-flow profile $a=500$

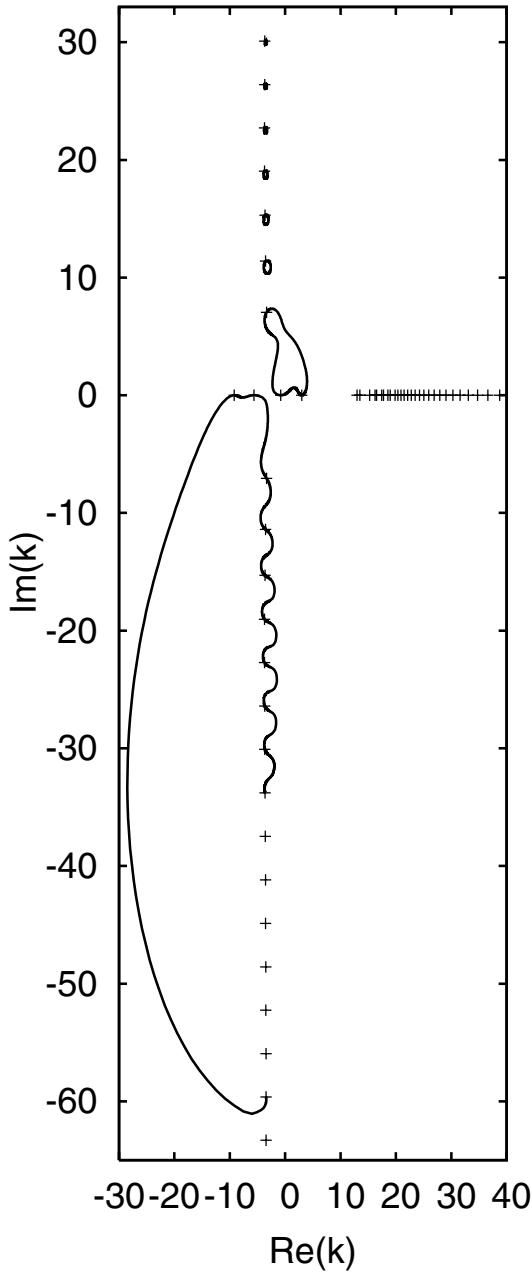

(c) mean-flow profile $a=50$

Figure 5. Trajectories of $\widetilde{k}$ for $\widetilde{\omega}=5, m=1, M_{\max }=0.5, Z_{d}=0.5+i X,-\infty<X<\infty$ : (a) - uniform mean flow, (b) - mean-flow velocity profile (85) with $a=500(\delta \approx 0.01 d)$, (c) - mean-flow velocity profile (85) with $a=50(\delta \approx 0.07 d)$. The hard wall eigenvalues are marked with the "+" sign.

uniform mean flow and two boundary-layer profiles (85) with $a=250$, and 50 are depicted. For the part of the acoustic spectrum shown in Figure 4 the quantity $\zeta_{1} \pi n / \kappa_{1} \approx 0.21 n$. Hence, with the exception of the first several indexes $n$ holds the relation $R \gg \zeta_{1} \pi n / \kappa_{1} \gg 1$. As a result, in agreement with the theoretical prediction (49), most of the lateral wavenumber trajectories are close to the circles whose centres are shifted slightly to the left from their hard-wall values in the upper half-plane $\widetilde{k}$ (positive $n$ ) and to the right in the lower half-plane (negative $n$ ). As predicted by the theory, for the uniform mean flow and the mean velocity profile with thin near-wall shear-flow sublayers $(a=250)$ the radii of these circles slowly grow with $n$ (i.e., away from the real axis). If the shear flow region is relatively thick, as is the case with $a=50$ in Figure 4c, opposite tendency prevails and the circles which are further away from the real axis have smaller radii, as in the zero mean flow case studied in Rienstra [16]. Note that the results depicted in Figure $4 \mathrm{c}$ for $a=50$, do not contradict the proposed theory, since the theory assumes that wall Mach number $M_{d}$ is non-zero. When the boundary-layer thickness is small, Ingard-Myers boundary condition permits the use of an "inviscid approximation" of the mean-flow profile with non-zero wall velocity instead of the "boundary-layer" mean-flow velocity profile which satisfies the no-slip condition. However, the range of validity of Ingard-Myers boundary condition is exceeded if the boundary layer becomes sufficiently thick. As a result, this places the restriction on the applicability of formula (49) for boundary-layer profiles. The example in Figure $4 \mathrm{c}$ shows that although Ingard-Myers boundary condition is correct in the limit of vanishing boundary-layer thickness, the numerical results based on its application 


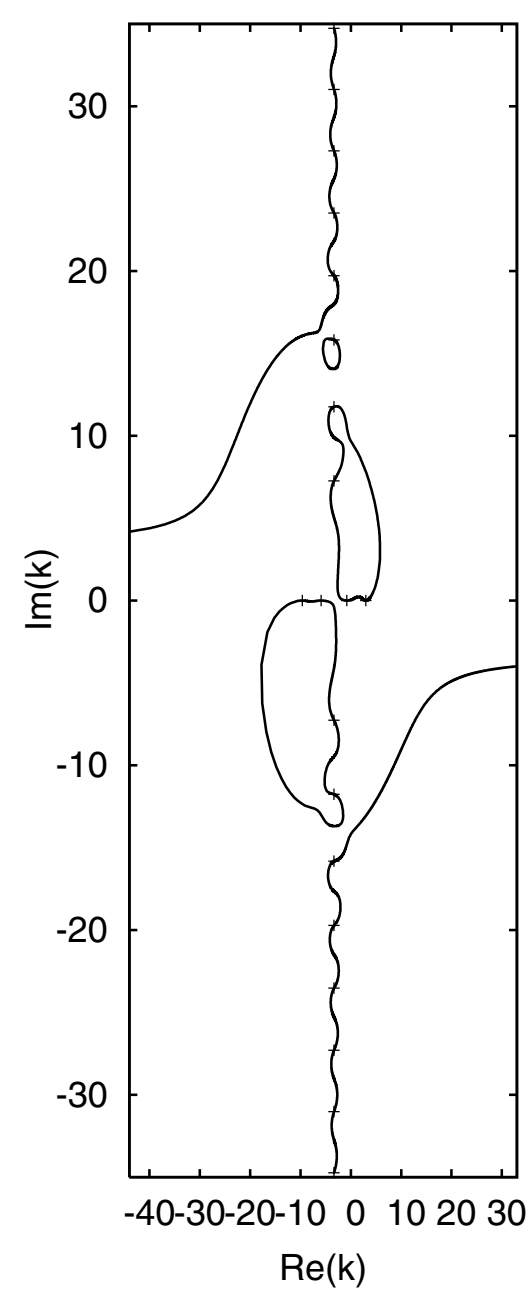

(a) uniform flow

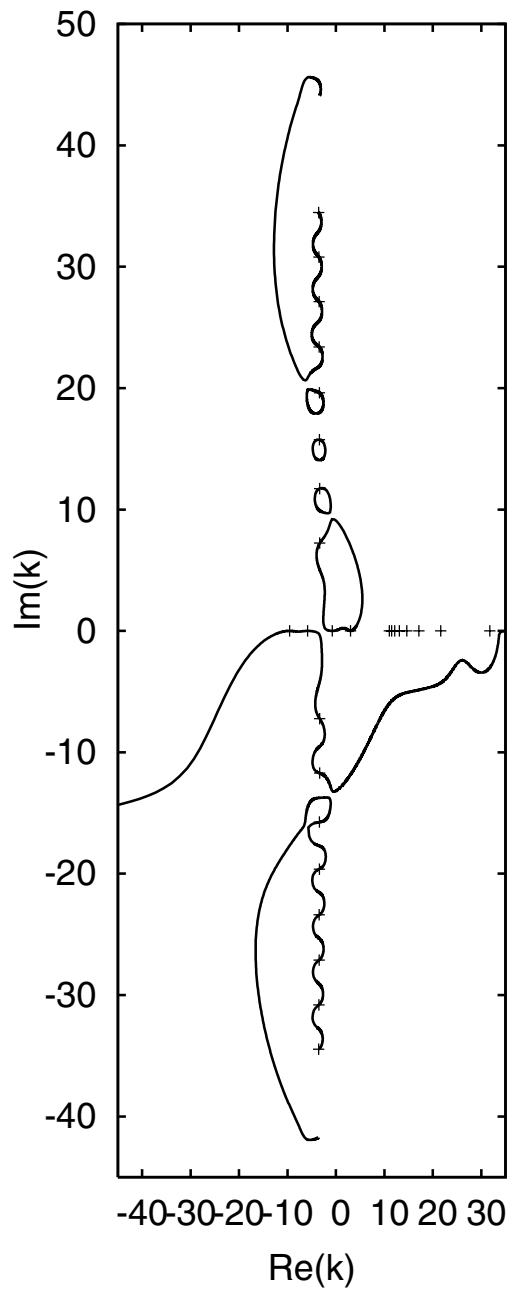

(b) mean-flow profile $a=500$



(c) mean-flow profile $a=50$

Figure 6. Trajectories of $\widetilde{k}$ for $\widetilde{\omega}=5, m=1, M_{\max }=0.5, Z_{d}=0.2+i X,-\infty<X<\infty$ : (a) - uniform mean flow, (b) - mean-flow velocity profile (85) with $a=500(\delta \approx 0.01 d)$, (c) - mean-flow velocity profile (85) with $a=50(\delta \approx 0.07 d)$. The hard wall eigenvalues are marked with the "+" sign.

can differ perceptibly from the results obtained for a mean-flow profile with vanishing wall velocity even when the boundary-layer thickness is as small as several percent of the free-field wavelength.

Figure 5 shows the trajectories followed by the lateral wavenumber $\widetilde{k}$ as $X$ varies from minus to plus infinity for $R=0.5$. Now in the uniform-flow case for almost all $n$ holds the inequality $\zeta_{1} \pi n / \kappa_{1} \gg R$. As a result, the number of circular trajectories reduces to only four and two slowly decaying vertical sinusoidal lines (55) and (56) can be observed in the upper and lower half-planes as $\operatorname{Im}(\widetilde{k}) \rightarrow \pm \infty$, respectively (Figure 5a). These lines smoothly merge with the horizontal surface-mode trajectories given by solution (58) which is valid when $\operatorname{Re}(\widetilde{k}) \rightarrow \pm \infty$. Estimates of the $\operatorname{Im}(\widetilde{k})$ for surface modes based on formulas (58) give an approximate value of \pm 8.67 which is reasonably close to the computed value of \pm 9.05 for large $\operatorname{Re}(\widetilde{k})$. However, already for the mean-flow velocity profile with a very thin near-wall sublayer shown in Figure $5 \mathrm{~b}(a=500)$ the eigenvalue pattern features substantial differences from its uniform-flow counterpart. It can be seen that only the lower surface-mode branch remains in Figure 5b. As opposed to the uniform-flow case, the imaginary part of the surface-mode eigenvalue does not remain finite for large $X$ but goes to zero. In the limit $X \rightarrow \infty$ the surface mode becomes a hydrodynamic mode which is localized near the duct wall and convected with almost zero phase velocity. As the boundary-layer thickness increases (Figure $5 \mathrm{c}$ ), the eigenvalue pattern deviates further away from its uniform-flow prototype and becomes structurally similar to the corresponding contour plot with zero mean-flow Mach number studied in Rienstra [16]. 
It is interesting to note that as opposed to the slowly-varying mean-flow profiles with non-zero wall Mach numbers studied in the previous section, the computations with the profiles of the boundary-layer type result in appearance of real-valued hydrodynamic modes, which number grows with mesh refinement. These modes can be seen in figures Figure $5 \mathrm{~b}$ and $5 \mathrm{c}$. Presently it is unclear whether the nature of these solutions is genuine or this is the feature of the numerical analysis.

Although further decrease of the real part of the wall impedance $R$ has little effect on lateral wavenumber contours for the uniform flow, the trajectories of $\widetilde{k}$ for the boundary-layer mean flow profiles undergo substantial structural changes as $R$ is reduced from 0.5 to 0.2 (see Figure 6). One remarkable feature of the eigenvalue patterns obtained for the boundary-layer type mean flow profiles and low $R$ is that as the imaginary part of the wall impedance $X$ varies from minus to plus infinity one of the lower half-plane cut-off acoustic modes gradually evolves into a hydrodynamic mode.

\section{Conclusions}

Overall, a robust numerical algorithm for determination of duct eigenmodes in sheared and/or swirling mean flows was developed. For sheared mean flows it was tested against existing data and was shown to be capable of handling very high frequencies.

An analytical theory was developed in order to check the proposed algorithm where the independent data was not available and to give insight into qualitative behaviour of the solutions.

The obtained results show that for the mean flow profiles with non-zero wall Mach numbers the number of hydrodynamic eigenmodes is finite and they are localized near the duct walls. For the boundary-layer mean-flow profiles which satisfy the no slip condition the situation is more complex. Our numerical analysis confirms the existence ${ }^{18}$ of a continuous, unbounded hydrodynamic spectrum which arises from the singularity in the equations where $\widetilde{\omega}-\widetilde{k} M(r)=0$. Further analysis of this result is needed.

\section{Acknowledgements}

This work was supported by the "Messiaen" European collaborative project (EU Technical Officer Dietrich Knörzer and Coordinator Jean-Louis Migeot, Free Field Technologies).

\section{References}

\footnotetext{
${ }^{1}$ Zorumski,W.E., 1974, Acoustic theory of axisymmetric multisectioned ducts. NASA TR R-419.

${ }^{2}$ Rienstra, S.W., 1999, Sound transmission in slowly varying circular and annular lined ducts with flow, J. Fluid Mech., vol. 380 , 279 - 296

${ }^{3}$ Rienstra, S.W., 2003, Sound propagation in slowly varying lined flow ducts of arbitrary cross-section, J. Fluid Mech., vol. 495,157 - 173

${ }^{4}$ Cooper, A. J., Peake, N., 2001, Propagation of unsteady disturbances in slowly varying duct with swirling mean flow. J. Fluid Mech., vol 445, pp.207-234.

${ }^{5}$ Eversman, W. 1991, Theoretical model for duct acoustic propagation and radiation. In Aeroacoustics of Flight Vehicles: Theory and Practice. Volume 2: Noise Control (Ed. H.H. Hubbard), chapter 13, pp.101-163.

${ }^{6}$ Golubev, V.V, Atassi, H.M., 1998, Acoustic vorticity waves in swirling flows. J. of Sound and Vibration, vol. 209(2), pp.203-222.

${ }^{7}$ Tam, C.K.W., Auriault, L., 1998, The wave modes in ducted swirling flows. J. Fluid Mech., vol. 371, pp.1-20.

${ }^{8}$ Nijboer, R. 2001, Eigenvalues and eigenfunctions of ducted swirling flows. AIAA 2001-2178, pp.1-11.

${ }^{9}$ Cooper, A.J., Peake, N., 2005, Upstream-radiated rotor-stator interaction noise in mean swirling flow. J. Fluid Mech., vol. 532, pp.219-250.

${ }^{10}$ Kousen, K.A. 1999, Eigenmodes of Ducted Flows With Radially-Dependent Axial and Swirl Velocity Components, NASA /CR-1999208881.

${ }^{11}$ Envia, E., 1998, A High Frequency Model of Cascade Noise, AIAA-98-2318, 4th AIAA/CEAS Aeroacoustics Conference, Toulouse, France, June 2-4, 1998.

${ }^{12}$ Pridmore-Brown, D. C., 1958, Sound Propagation in a Fluid Flowing through an attenuating Duct, Journal of Fluid Mechanics, 4, pp. 393-406.

${ }^{13}$ Ingard, K.U., 1959, Influence of fluid motion past a plane boundary on sound reflection, absorption and transmission. Journal of the Acoustical Society of America. 31(7), pp.1035-1036.

${ }^{14}$ Myers, M.K., 1980, On the acoustic boundary condition in the presence of flow. Journal of Sound and Vibration. 71(3), pp. 429-434.

${ }^{15}$ Barantsev, R.G., Engelgart, V.N., 1987, Asymptotic methods in gas and fluid dynamics. Leningrad University Publishers. pp.33-40.

${ }^{16}$ Rienstra, S.W. 2003, A classification of duct modes based on surface waves. Wave Motion. Elsevier. vol. 37 pp.119-135.

${ }^{17}$ Fornberg,B., Driscoll, T.A., 1999, A fast spectral algorithm for nonlinear wave equations with linear dispersion. J. Comput. Phys. vol. 155, pp.456-467.

${ }^{18}$ Drazin, P.G., Reid, W.H., 2004, Hydrodynamic Stability, Cambridge University Press, 2nd edition, Cambridge.
} 